\title{
Metabolic modelling of polyhydroxyalkanoate copolymers production by mixed microbial cultures João ML Dias ${ }^{\dagger}$, Adrian Oehmen ${ }^{\dagger}$, Luísa S Serafim ${ }^{\dagger}$, Paulo C Lemos ${ }^{\dagger}$, Maria AM Reis ${ }^{\dagger}$ and Rui Oliveira*
}

Address: REQUIMTE, Chemistry Department, FCT/Universidade Nova de Lisboa, 2829-516 Caparica, Portugal

Email: João ML Dias - joao.dias@dq.fct.unl.pt; Adrian Oehmen - adriano@dq.fct.unl.pt; Luísa S Serafim - luisa.serafim@dq.fct.unl.pt; Paulo C Lemos - pac@fct.unl.pt; Maria AM Reis - amr@dq.fct.unl.pt; Rui Oliveira* - rui.oliveira@dq.fct.unl.pt

${ }^{*}$ Corresponding author †Equal contributors

Published: 8 July 2008

BMC Systems Biology 2008, 2:59 doi:10.1 186/1752-0509-2-59
Received: 31 January 2008

Accepted: 8 July 2008

This article is available from: http://www.biomedcentral.com/I752-0509/2/59

(c) 2008 Dias et al; licensee BioMed Central Ltd.

This is an Open Access article distributed under the terms of the Creative Commons Attribution License (http://creativecommons.org/licenses/by/2.0), which permits unrestricted use, distribution, and reproduction in any medium, provided the original work is properly cited.

\begin{abstract}
Background: This paper presents a metabolic model describing the production of polyhydroxyalkanoate (PHA) copolymers in mixed microbial cultures, using mixtures of acetic and propionic acid as carbon source material. Material and energetic balances were established on the basis of previously elucidated metabolic pathways. Equations were derived for the theoretical yields for cell growth and PHA production on mixtures of acetic and propionic acid as functions of the oxidative phosphorylation efficiency, P/O ratio. The oxidative phosphorylation efficiency was estimated from rate measurements, which in turn allowed the estimation of the theoretical yield coefficients.
\end{abstract}

Results: The model was validated with experimental data collected in a sequencing batch reactor (SBR) operated under varying feeding conditions: feeding of acetic and propionic acid separately (control experiments), and the feeding of acetic and propionic acid simultaneously. Two different feast and famine culture enrichment strategies were studied: (i) either with acetate or (ii) with propionate as carbon source material. Metabolic flux analysis (MFA) was performed for the different feeding conditions and culture enrichment strategies. Flux balance analysis (FBA) was used to calculate optimal feeding scenarios for high quality PHA polymers production, where it was found that a suitable polymer would be obtained when acetate is fed in excess and the feeding rate of propionate is limited to $\sim 0.17 \mathrm{C}-\mathrm{mol} /(\mathrm{C}-\mathrm{mol} . \mathrm{h})$. The results were compared with published pure culture metabolic studies.

Conclusion: Acetate was more conducive toward the enrichment of a microbial culture with higher PHA storage fluxes and yields as compared to propionate. The P/O ratio was not only influenced by the selected microbial culture, but also by the carbon substrate fed to each culture, where higher P/O ratio values were consistently observed for acetate than propionate. MFA studies suggest that when mixtures of acetate and propionate are fed to the cultures, the catabolic activity is primarily guaranteed through acetate uptake, and the characteristic $\mathrm{P} / \mathrm{O}$ ratio of acetate prevails over that of propionate. This study suggests that the PHA production process by mixed microbial cultures has the potential to be comparable or even more favourable than pure cultures. 


\section{Background}

Polyhydroxyalkanoates are biopolymers with physicochemical properties similar to polypropylene but with the advantage of being biodegradable and biocompatible. Industrial PHA production technology is currently based on bacteria cultivation using pure cultures grown in welldefined nutrient deficient synthetic media with single substrates [1]. The relatively high production costs constitute presently the most important barrier to PHA becoming a commodity polymer in direct competition with oilbased polymers. The cost of the substrate and of the equipment required for aseptic operation is responsible for about $40 \%$ of the total PHA production cost [1].

Potential strategies for reducing both the operational and capital expenses of PHA production are the use of open mixed cultures of microorganisms instead of pure cultures, and the use of waste materials as the carbon substrate. Activated sludge is known to accumulate up to $65 \%$ PHA per cell dry weight [2-4]. There are currently many possibilities of industrial and agricultural wastes that could be directed to the production of PHA on the basis of open mixed microbial systems $[5,6]$.

Mixed microbial cultures cannot directly metabolize sugars into PHA. A pre-fermentation step is required in order to convert sugars into volatile fatty acids (VFA) such as acetic, propionic, valeric and butyric acid. This pre-fermentation step can, however, be accomplished with high yield $[5,7]$. The final PHA molecular structure is highly dependent on the VFA composition used as carbon source. The most well-studied case is the production of polyhydroxybutyrate (PHB), a homopolymer, obtained when acetic acid is used as the sole carbon source. The metabolism for this transformation (included in Figure 1) can be defined by 6 basic reactions $[8,9]$ : acetate uptake $\left(\mathrm{R}_{1}\right)$, growth on acetyl-CoA $\left(\mathrm{R}_{4}\right)$, catabolism $\left(\mathrm{R}_{6}\right)$, oxidative phosphorylation $\left(R_{8}\right)$, maintenance $\left(R_{7}\right)$ and PHB production $\left(\mathrm{R}_{\mathrm{PHB}}\right)$. A metabolic model based on these reactions was comprehensively validated with experimental data [10]. This study demonstrated an excellent agreement between the model predictions and experimental data.

The properties and quality of PHA are highly dependent on the monomeric composition, which, in turn, is dependent on the VFA mixture adopted as carbon source. PHB is a relatively low quality polymer since it is too brittle for many applications $[1,11]$. In terms of PHA processing, the production of copolymers appears to be of higher commercial interest [12-14]. The production of PHB homopolymer and copolymers of 3-hydroxybutyrate (3HB), 3-hydroxyvalerate (3HV) and 3-hydroxy-2-methylvalerate $(3 \mathrm{H} 2 \mathrm{MV})$ from mixtures of acetic and propionic acid has been studied by several researchers $[11,15-$
17]. There are, however, no metabolic models currently available describing the production of such copolymers. Metabolic models can be used to facilitate process optimization, and also serve as a reference basis in the interpretation of data arising from the study of biological processes. The comparison of experimentally-determined stoichiometry with the theoretical model predictions allows a better understanding of the processes under study. The main objective of this work was to extend existing PHB models $[8,10]$ to the production of PHA copolymers from mixtures of VFA. This study will be focused on the PHA production phase, and is confined to mixtures of acetic and propionic acid, which are seen as the most promising for the synthesis of high quality PHAs $[11,18]$.

\section{Metabolic model}

A schematic of the metabolic model developed in this study is shown in Figure 1. Acetate and propionate are taken up inside the cell, and used for PHA production and biomass growth. Energy is generated within the cells through oxidative phosphorylation, while a portion of this energy is also necessary for cell maintenance. A detailed description of the biochemical reactions, material and energy balances, kinetic reactions and metabolic fluxes is provided below.

\section{Basic reactions}

The detailed biochemical reactions involved in the PHA production process using acetate and propionate carbon sources under aerobic conditions are shown in Table 1. All reactions are expressed on a carbon-mole basis. In the feast phase, acetate and propionate are taken up inside the cells by means of active transport, requiring one mole of ATP per mole of carbon source. They are then converted into acetyl-CoA and propionyl-CoA, respectively, at the cost of another mole of ATP per mole of acetate or propionate. The net reactions are shown in $R_{1}$ and $R_{2}$ [19].

While acetate is converted to acetyl-CoA and propionylCoA is produced from propionate, it has also been observed that a portion of the propionyl-CoA produced from propionate uptake is converted into acetyl-CoA [11]. This transformation may potentially proceed through 5 different biochemical pathways [11], however, the net material and reducing power transformations are identical through each pathway. The difference between the pathways lies only in the amount of energy generated. For the purposes of this model, it was assumed that propionyl-CoA was first converted to succinyl-CoA via the methylmalonyl-CoA pathway, then converted to oxaloacetate, pyruvate and acetyl-CoA. The net reaction is described in $\mathrm{R}_{3}$.

In PHA production, acetyl-CoA and propionyl-CoA are reduced and condensed to produce a polymer consisting 


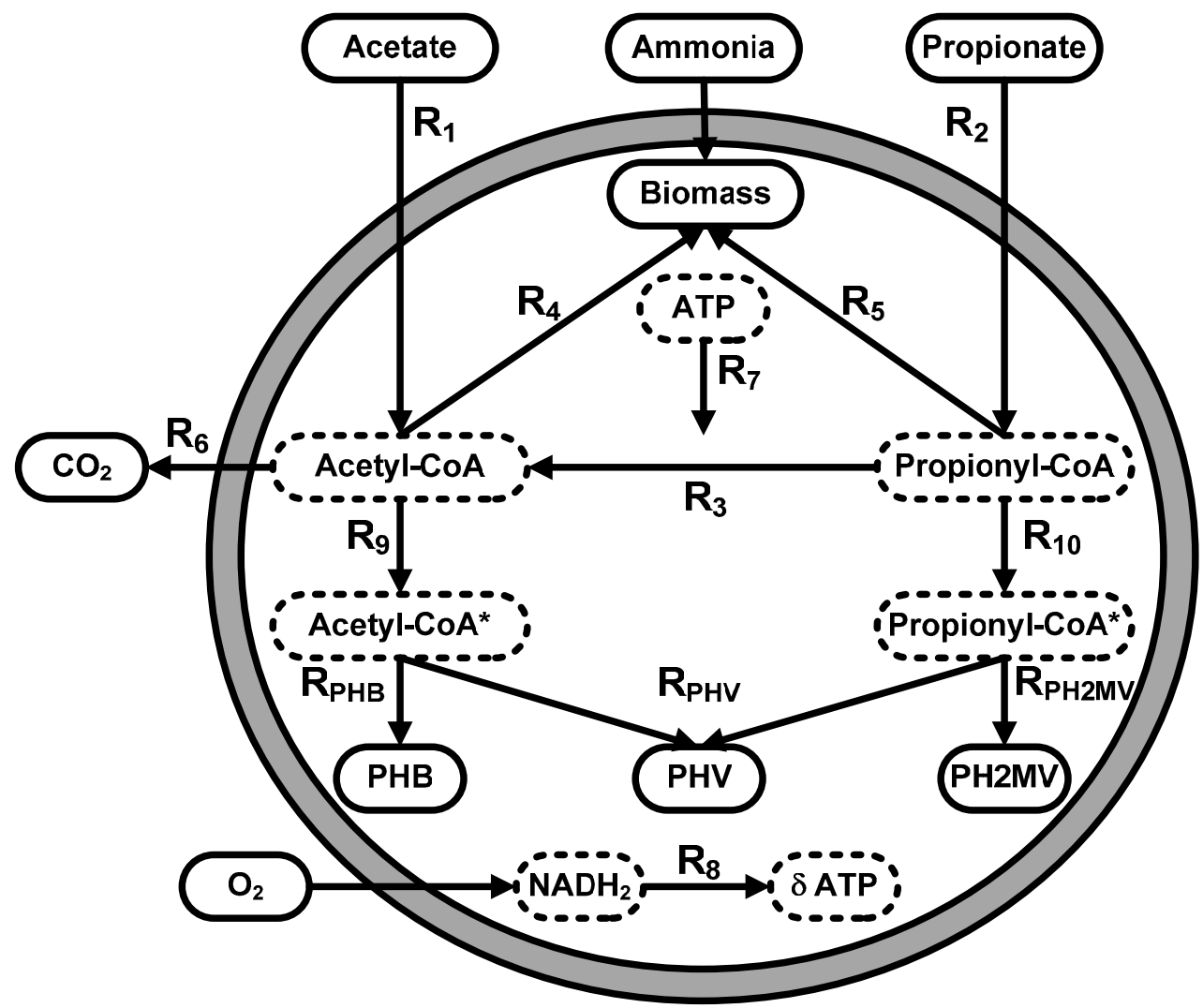

Figure I

PHA metabolic network. Figure shows schematic representation of the metabolic reactions, substrates, products (both marked by full lines) and metabolites (marked by dashed lines) involved in PHA production by mixed microbial cultures.

of various 3-hydroxyalkanoates (3HAs) monomers. 3HB is formed from 2 acetyl-CoA monomers, one acetyl-CoA and one propionyl-CoA combine to form either $3 \mathrm{HV}$ or 3hydroxy-2-methylbutyrate $(3 \mathrm{H} 2 \mathrm{MB})$ (which are isomers of each other, and henceforth expressed only as $3 \mathrm{HV}$, indicating the sum of these 2 compounds), while 2 propionylCoA monomers form $3 \mathrm{H} 2 \mathrm{MV}$. The fraction of each PHA monomer produced by the cells depends on the fluxes of acetyl-CoA and propionyl-CoA available for polymer production. It also depends on whether the cells tend to reduce and condense acetyl-CoA and propionyl-CoA in a random fashion, or whether preferential binding occurs between e.g. acetyl-CoA and propionyl-CoA. For the purposes of metabolic model development, however, it is necessary to express each reaction in relation to the overall mass, energy and redox transformations. Thus, it is more convenient to express the various potential PHA fractions produced by the cells in terms of the reduced and condensed monomers produced from acetyl-CoA or propionyl-CoA. As shown in $\mathrm{R}_{9}$ and $\mathrm{R}_{10}$, these monomers are represented as acetyl-CoA* and propionyl-CoA*, respectively, in a similar fashion as expressed by a previous study [20]. The PHA formation process from acetyl-CoA* and
propionyl-CoA* has no further demand for energy or reducing power.

Biomass growth occurs from acetyl-CoA and propionylCoA, respectively. The energy required for the production of $1 \mathrm{C}$-mol of biomass from acetyl-CoA is represented as $\mathrm{K}_{1}$, which has been estimated as $1.7 \mathrm{~mol}$ ATP [21]. The reaction of biomass growth from acetyl-CoA was determined through stoichiometric and redox balancing, and is shown in $\mathrm{R}_{4}$. The amount of energy required for biomass synthesis from propionyl-CoA is represented as $\mathrm{K}_{2}$, which has been estimated to be $1.38 \mathrm{~mol}$ ATP per C-mol of biomass formed [22]. Similarly, it was assumed that propionyl-CoA is converted to succinyl-CoA for biomass synthesis [22]. The net reaction is shown in $R_{5}$. The biomass formula was assumed to be $\mathrm{CH}_{1.4} \mathrm{O}_{0.4} \mathrm{~N}_{0.2}$ [23].

Catabolism of acetyl-CoA and propionyl-CoA also occurs in the microbial cells. Acetyl-CoA is converted to $\mathrm{CO}_{2}$ through the tricarboxylic acid cycle (TCA) as described in $\mathrm{R}_{6}$ [19]. The catabolism of propionyl-CoA is assumed to occur via acetyl-CoA, where $R_{3}$ is followed by $R_{6}$. 
Table I: Metabolic model of PHA production from mixtures of acetate and propionate by mixed microbial cultures

\begin{tabular}{|c|c|}
\hline Reaction & Stoichiometry \\
\hline $\mathbf{R}_{1}$ : Acetate Uptake & $\mathrm{CH}_{2} \mathrm{O}+\mathrm{ATP} \rightarrow \mathrm{CHO}_{0.5}+0.5 \cdot \mathrm{H}_{2} \mathrm{O}$ \\
\hline $\mathbf{R}_{2}:$ Propionate Uptake & $\mathrm{CH}_{2} \mathrm{O}_{2 / 3}+0.67 \cdot \mathrm{ATP} \rightarrow \mathrm{CH}_{4 / 3} \mathrm{O}_{1 / 3}+0.33 \cdot \mathrm{H}_{2} \mathrm{O}$ \\
\hline $\mathbf{R}_{3}$ : Propionyl-CoA converted to Acetyl-CoA & I.5. $\mathrm{CH}_{4 / 3} \mathrm{O}_{1 / 3}+\mathrm{H}_{2} \mathrm{O} \rightarrow \mathrm{CHO}_{0.5}+1.5 \cdot \mathrm{NADH}_{2}+0.5 \cdot \mathrm{CO}_{2}$ \\
\hline $\mathbf{R}_{4}:$ Growth on Acetyl-CoA & I.27 $\mathrm{CHO}_{0.5}+0.2 \cdot \mathrm{NH}_{3}+\mathrm{K} \cdot \cdot \mathrm{ATP}+0.3 \cdot \mathrm{H}_{2} \mathrm{O} \rightarrow \mathrm{CH}_{1.4} \mathrm{~N}_{0.2} \mathrm{O}_{0.4}+0.53 \cdot \mathrm{NADH}_{2}+0.27 \cdot \mathrm{CO}_{2}$ \\
\hline \multirow[t]{2}{*}{$\mathbf{R}_{5}:$ Growth on Propionyl-CoA } & I.06 $\mathrm{CH}_{4 / 3} \mathrm{O}_{1 / 3}+0.2 \cdot \mathrm{NH}_{3}+\mathrm{K}_{2} \cdot \mathrm{ATP}+0.17 \cdot \mathrm{H}_{2} \mathrm{O} \rightarrow \mathrm{CH}_{1.4} \mathrm{~N}_{0.2} \mathrm{O}_{0.4}+0.47 \cdot \mathrm{NADH}_{2}+$ \\
\hline & $0.06 \cdot \mathrm{CO}_{2}$ \\
\hline $\mathbf{R}_{6}$ : Catabolism & $\mathrm{CHO}_{0.5}+1.5 \cdot \mathrm{H}_{2} \mathrm{O} \rightarrow \mathrm{CO}_{2}+2 \cdot \mathrm{NADH}_{2}+0.5 \cdot \mathrm{ATP}$ \\
\hline $\mathbf{R}_{\mathbf{7}}:$ Maintenance & ATP $\rightarrow \mathrm{m}_{\text {ATP }}$ \\
\hline $\mathbf{R}_{\mathbf{8}}$ : Oxidative Phosphorylation & $\mathrm{NADH}_{2}+0.5 \cdot \mathrm{O}_{2} \rightarrow \mathrm{H}_{2} \mathrm{O}+\delta \cdot \mathrm{ATP}$ \\
\hline $\mathbf{R}_{\mathbf{9}}$ : Acetyl-CoA* Production & $\mathrm{CHO}_{0.5}+0.25 \cdot \mathrm{NADH}_{2} \rightarrow \mathrm{CH}_{1.5} \mathrm{O}_{0.5}$ \\
\hline
\end{tabular}

Energy in the form of ATP is produced from $\mathrm{NADH}_{2}$ through oxidative phosphorylation. The amount of ATP generated per mole of $\mathrm{NADH}_{2}$ oxidized is expressed by the $\mathrm{P} / \mathrm{O}$ ratio, $\delta$, which represents the efficiency of oxidative phosphorylation [24]. This reaction is expressed in $R_{8}$.

In addition to the energetic transformations described above, energy is also required for cell maintenance. The rate of ATP consumption for maintenance purposes is described in $\mathrm{R}_{7}$ as $\mathrm{m}_{\text {ATP }}$.

\section{Pseudo steady-state material balancing of intracellular intermediates}

The metabolic network of Figure 1 has $q=13$ metabolic reactions, $m=6$ intracellular metabolites (marked by dashed lines), 4 input substrates (acetic and propionic acid, ammonia and oxygen) and 5 end-products [biomass, PHB, poly(3-hydroxyvalerate) (PHV), poly(2methyl-3-hydroxyvalerate) (PH2MV) and $\left.\mathrm{CO}_{2}\right]$. The steady state material balances to the $m$ intracellular intermediates (acetyl-CoA, acetyl-CoA*, propionyl-CoA, propionyl-CoA*, $\mathrm{NADH}_{2}$ and ATP) are:

$$
\begin{gathered}
\text { Ac-CoA: } R_{1}+R_{3}-1.27 \cdot R_{4}-R_{6}-R_{9}=0 \\
\text { Ac-CoA*: } R_{9}-R_{P H B}-0.4 \cdot R_{P H V}=0 \\
\text { Prop-CoA: } R_{2}-1.5 \cdot R_{3}-1.06 \cdot R_{5}-R_{10}=0 \\
\text { Prop-CoA*: } R_{10}-0.6 \cdot R_{P H V}-R_{P H 2 M V}=0
\end{gathered}
$$

$\mathrm{NADH}_{2}: 1.5 \cdot R_{3}+0.53 \cdot R_{4}+0.47 \cdot R_{5}+2 \cdot R_{6}-R_{8}-0.25 \cdot R_{9}$ $-0.17 \cdot R_{10}=0$

ATP: $-R_{1}-0.67 \cdot R_{2}-K_{1} \cdot R_{4}-K_{2} \cdot R_{5}+0.5 \cdot R_{6}-R_{7}+\delta \cdot R_{8}=0$

\section{Constraints to the metabolic network}

From acetyl-CoA* and propionyl-CoA*, the resulting PHA polymer can be comprised of either PHB (2 acetyl$\mathrm{CoA}^{*}$ molecules), PHV (one acetyl-CoA* and one propionyl-CoA*) and PH2MV (2 propionyl-CoA*). The PHA composition depends on whether selective or random condensation of acetyl-CoA* and propionyl-CoA* takes place. In the propionate enriched culture selective condensation was observed, where acetyl-CoA* preferentially bounds with propionyl-CoA*, forming PHV [11]. Since acetyl-CoA* was generated in higher abundance than propionyl-CoA*, the remaining acetyl-CoA* condensed to form $\mathrm{PHB}$, while $\mathrm{PH} 2 \mathrm{MV}$ was not produced by the sludge. This selective condensation is expressed by the following three additional equations:

$$
\begin{gathered}
R_{P H B}=R_{9}-\frac{2}{3} \cdot R_{10} \\
R_{P H V}=\frac{5}{3} \cdot R_{10} \\
R_{P H 2 M V}=0
\end{gathered}
$$

since $R_{P H 2 M V}=0$ (Eq. 2c), Eqs. (2a-b) are linearly dependent to Eqs. (1a-f), only a single additional constraint is introduced with $\mathrm{R}_{\mathrm{PH} 2 \mathrm{MV}}=0$.

In the acetate enriched culture, the PHA composition consisted of a lower PHV fraction and a higher PH2MV and $\mathrm{PHB}$ fraction, as is expected by microorganisms performing random condensation of acetyl-CoA* and propionyl$\mathrm{CoA}^{*}$. In this latter case the following three equations are applied [20]:

$$
R_{P H B}=\frac{2 \cdot R_{9}^{2}}{2 \cdot R_{9}+3 \cdot R_{10}}
$$


Table I: Metabolic model of PHA production from mixtures of acetate and propionate by mixed microbial cultures (Continued)

\begin{tabular}{lc}
\hline $\mathbf{R}_{10}:$ Propionyl-CoA* Production & $\mathrm{CH}_{4 / 3} \mathrm{O}_{1 / 3}+0.17 \cdot \mathrm{NADH}_{2} \rightarrow \mathrm{CH}_{5 / 3} \mathrm{O}_{1 / 3}$ \\
$\mathbf{R}_{\mathbf{P H B}}:$ PHB Production & $\mathrm{CH}_{1.5} \mathrm{O}_{0.5} \rightarrow \mathrm{PHB}$ \\
$\mathbf{R}_{\mathbf{P H V}}$ : PHV Production & $0.4 \cdot \mathrm{CH}_{1.5} \mathrm{O}_{0.5}+0.6 \cdot \mathrm{CH}_{5 / 3} \mathrm{O}_{1 / 3} \rightarrow \mathrm{PHV}$ \\
$\mathbf{R}_{\mathbf{P H} 2 \mathrm{MV}}:$ PH2MV Production & $\mathrm{CH}_{5 / 3} \mathrm{O}_{1 / 3} \rightarrow \mathrm{PH} 2 \mathrm{MV}$
\end{tabular}

$$
\begin{gathered}
R_{P H V}=\frac{5 \cdot R_{9} \cdot R_{10}}{2 \cdot R_{9}+3 \cdot R_{10}} \\
R_{P H 2 M V}=\frac{3 \cdot R_{10}^{2}}{2 \cdot R_{9}+3 \cdot R_{10}}
\end{gathered}
$$

The $R_{9}$ and $R_{10}$ fluxes are sufficient to calculate the fluxes of $R_{P H B^{\prime}} R_{P H V}$ and $R_{P H 2 M V}$.

On the other hand, constraints ( $3 a-c)$ automatically obey the material balances (1a-f) (see [20] for details), thus also a single additional constraint can also be added to the material balances here ( $1 \mathrm{a}-\mathrm{f})$.

\section{Dynamic material balancing of substrates and end- products}

The transient material balances in a batch system of input substrates (acetate, propionate, active biomass, ammonia) and intracellular contents of $\mathrm{PHB}, \mathrm{PHV}$ and $\mathrm{PH} 2 \mathrm{MV}$ are the following:

$$
\begin{gathered}
\frac{d A c}{d t}=-R_{1} \cdot X \\
\frac{d \operatorname{Pr} o p}{d t}=-R_{2} \cdot X \\
\frac{d N}{d t}=-0.2 \cdot\left(R_{4}+R_{5}\right) \cdot X \\
\frac{d X}{d t}=\left(R_{4}+R_{5}\right) \cdot X \\
\frac{d f_{P H B}}{d t}=R_{P H B}-\left(R_{4}+R_{5}\right) \cdot f_{P H B} \\
\frac{d f_{P H V}}{d t}=R_{P H V}-\left(R_{4}+R_{5}\right) \cdot f_{P H V} \\
\frac{d f_{P H 2 M V}}{d t}=R_{P H 2 M V}-\left(R_{4}+R_{5}\right) \cdot f_{P H 2 M V}
\end{gathered}
$$

\section{Theoretical yields}

The theoretical yields and VFA and oxygen maintenance coefficients were derived analytically from the material balance equations ( $1 \mathrm{a}-\mathrm{f})$. The expressions are compiled in Table 2 . The theoretical yield coefficients are functions of $\delta, K_{1}, K_{2}$ and $y$, the ratio of propionate flux to total carbon flux (see Eq. 5):

$$
y=\frac{R_{2}}{R_{1}+R_{2}}
$$

\section{Kinetic model}

As stated above, the metabolic network has $q=13$ fluxes and a total number of 7 constraints. Therefore, at least 6 fluxes must be defined a priori in order to calculate the network fluxes. Table 3 compiles the kinetic equations used in this work to model the missing fluxes. This model is an extension of a previous acetate to PHB metabolic model [10]. Briefly, the model defines the following kinetic rates:

- Growth on acetyl-CoA $\left(R_{4}\right)$ is limited by the concentrations of acetate and ammonia.

- Growth on propionyl-CoA $\left(R_{5}\right)$ is limited by the concentrations of propionate and ammonia.

- Catabolism $\left(R_{6}\right)$ is limited by total VFA concentration. The kinetic equation for catabolism is only used for mixtures of acetate and propionate to fulfil the 6 fluxes required to perform MFA.

- Maintenance $\left(R_{7}\right)$ is limited by total VFA concentration.

- Acetyl-CoA* synthesis $\left(R_{9}\right)$ is limited by total VFA concentration and inhibited by the intracellular PHA content. Intracellular PHA inhibition has been validated experimentally by several authors [8-10,25-27].

- Propionyl-CoA* synthesis $\left(R_{10}\right)$ is limited by propionate concentration and inhibited by the intracellular PHA content. 
Table 2: Theoretical yields and maintenance coefficients

\begin{tabular}{|c|c|c|}
\hline Process description & VFA & Oxygen \\
\hline \multicolumn{3}{|l|}{ Cell growth on acetate } \\
\hline & $Y_{X, A c / S}=\frac{(4 \cdot \delta+0.67 \cdot y \cdot \delta+0.33 \cdot y-1)}{\left(4 \cdot \delta+2 \cdot K_{1}+1.27\right)}$ & $Y_{X, A c / O 2}=\frac{(4 \cdot \delta+0.67 \cdot y \cdot \delta+0.33 \cdot y-1)}{\left(0.33 \cdot y \cdot K_{1}-0.12 \cdot \gamma+2.27+2 \cdot K_{1}\right)}$ \\
\hline \multicolumn{3}{|l|}{ Cell growth on propionate } \\
\hline & $Y_{X, \operatorname{Pr} o p / S}=\frac{(4 \cdot \delta+0.67 \cdot y \cdot \delta+0.33 \cdot y-1)}{\left(4 \cdot \delta+2 \cdot K_{2}+0.71\right)}$ & $Y_{X, \operatorname{Pr} o p / O 2}=\frac{(4 \cdot \delta+0.67 \cdot y \cdot \delta+0.33 \cdot y-1)}{\left(0.33 \cdot y \cdot K_{2}-0.22 \cdot y+1.71+2 \cdot K_{2}\right)}$ \\
\hline \multicolumn{3}{|l|}{ PHB Storage } \\
\hline & $Y_{P H B / S}=\frac{(4 \cdot \delta+0.67 \cdot \gamma \cdot \delta+0.33 \cdot y-1)}{(4 \cdot 5 \cdot \delta+1)}$ & $Y_{\mathrm{PHB} / \mathrm{O} 2}=\frac{(4 \cdot \delta+0.67 \cdot \gamma \cdot \delta+0.33 \cdot \mathrm{y}-1)}{(2.12-0.21 \cdot \gamma)}$ \\
\hline \multicolumn{3}{|l|}{ PHV Storage } \\
\hline & $Y_{P H V / S}=\frac{(4 \cdot \delta+0.67 \cdot y \cdot \delta+0.33 \cdot y-1)}{(4 \cdot 8 \cdot \delta+0.8)}$ & $Y_{\mathrm{PHV} / \mathrm{O} 2}=\frac{(4 \cdot \delta+0.67 \cdot \gamma \cdot \delta+0.33 \cdot \mathrm{y}-1)}{(2-0.27 \cdot \gamma)}$ \\
\hline \multicolumn{3}{|l|}{ PH2MV Storage } \\
\hline & $Y_{P H 2 M V / S}=\frac{(4 \cdot \delta+0.67 \cdot \gamma \cdot \delta+0.33 \cdot \mathrm{y}-1)}{(5 \cdot \delta+0.67)}$ & $Y_{\mathrm{PH} 2 \mathrm{MV} / \mathrm{O} 2}=\frac{(4 \cdot \delta+0.67 \cdot y \cdot \delta+0.33 \cdot \mathrm{y}-1)}{(1.92-0.31 \cdot y)}$ \\
\hline \multicolumn{3}{|l|}{ Maintenance } \\
\hline & $m_{S}=\frac{2}{(4 \cdot \delta+0.67 \cdot y \cdot \delta+0.33 \cdot y-1)} \cdot m_{A T P}$ & $m_{\mathrm{O} 2}=\frac{(2+0.33 \cdot y)}{(4 \cdot \delta+0.67 \cdot y \cdot \delta+0.33 \cdot \mathrm{y}-1)} \cdot m_{A T P}$ \\
\hline
\end{tabular}

\section{Methods}

Reactors operation

In this study, two SBR for the production of PHA, were operated over four years. The sludge in each reactor was adapted to either acetate or propionate as the sole carbon source. The reactors working volume was 1 litre and the total SBR cycle duration was $12 \mathrm{~h}$, consisting of $10.5 \mathrm{~h}$ of aerobiosis, $1 \mathrm{~h}$ of settling (agitation and air sparging switched off) and $0.5 \mathrm{~h}$ to withdraw half of the volume, which was replaced by the same volume of fresh medium during the first $15 \mathrm{~min}$ at the beginning of the next cycle. The hydraulic retention time (HRT) was therefore 1 day. At the end of each cycle, before settling, a defined volume of biomass was removed to keep the sludge retention time

Table 3: Kinetic model

Reaction

Kinetics

\section{$\mathbf{R}_{4}$ : Growth on Acetyl-CoA}

$\mathbf{R}_{5}$ : Growth on Propionyl-CoA

$$
R_{4}=R_{4, \max } \cdot \frac{A c}{A c+K_{S}} \cdot \frac{N}{N+K_{N}}
$$

\section{$\mathbf{R}_{6}$ : Catabolism}

$$
R_{5}=R_{5, \max } \cdot \frac{\operatorname{Pr} o p}{\operatorname{Pr} o p+K_{S}} \cdot \frac{N}{N+K_{N}}
$$

$\mathbf{R}_{7}$ : Maintenance

$$
R_{6}=R_{6, \max } \cdot \frac{S}{S+K_{S}}
$$

\section{$\mathbf{R}_{\mathbf{9}}$ : Acetyl-CoA* Production}

$$
R_{7}=R_{7, \max } \cdot \frac{S}{S+K S}
$$

$$
R_{9}=\max \left\{R_{9, \max } \cdot \frac{S}{S+K_{S}} \cdot\left[1-\left(\frac{f_{P H A}}{f_{P H A, \max }}\right)^{\alpha}\right], \frac{2}{3} \cdot R_{10}\right\}
$$

$\mathbf{R}_{10}$ : Propionyl-CoA* Production

$$
R_{10}=R_{10, \text { max }} \cdot \frac{S}{S+K_{S}} \cdot\left[1-\left(\frac{f_{P H A}}{f_{P H A, \max }}\right)^{\alpha}\right]
$$


(SRT) at 10 days. Oxygen was supplied by an air compressor through a ceramic membrane disperser introduced inside the reactor at an airflow rate of $1.0 \mathrm{vvm}$ (volume air/(volume reactor.min)), allowing for the dissolved oxygen (DO) concentration to be around $80 \%$ of the saturation value. The reactors were operated without $\mathrm{pH}$ control but its value was monitored on-line and ranged between 8.0 and 9.2; the temperature was controlled at $22^{\circ} \mathrm{C}$ and the stirring rate at $250 \mathrm{rpm}$.

\section{Culture medium}

The standard medium used in the SBRs was composed of (per litre of distilled water): $1.269 \mathrm{~g} \mathrm{CH}_{3} \mathrm{CH}_{2} \mathrm{COOH}$ or $4.0796 \mathrm{~g}$ of $\mathrm{CH}_{3} \mathrm{COONa} .3 \mathrm{H}_{2} \mathrm{O}, 600 \mathrm{mg} \mathrm{MgSO}_{4} .7 \mathrm{H}_{2} \mathrm{O}$, $160 \mathrm{mg} \mathrm{NH}{ }_{4} \mathrm{Cl}, 100 \mathrm{mg}$ EDTA, $92 \mathrm{mg} \mathrm{K} \mathrm{HPO}_{4}, 45 \mathrm{mg}$ $\mathrm{KH}_{2} \mathrm{PO}_{4}, 70 \mathrm{mg} \mathrm{CaCl} 2.2 \mathrm{H}_{2} \mathrm{O}$ and $2 \mathrm{ml}$ of trace elements solution. The trace solution consisted of (per litre of distilled water): $1500 \mathrm{mg} \mathrm{FeCl} \cdot 6 \mathrm{H}_{2} \mathrm{O}, 150 \mathrm{mg} \mathrm{H} \mathrm{BO}_{3}, 150$ $\mathrm{mg} \mathrm{CoCl}_{2} \cdot 6 \mathrm{H}_{2} \mathrm{O}, 120 \mathrm{mg} \mathrm{MnCl}_{2} \cdot 4 \mathrm{H}_{2} \mathrm{O}, 120 \mathrm{mg}$ $\mathrm{ZnSO}_{4} \cdot 7 \mathrm{H}_{2} \mathrm{O}, \quad 60 \mathrm{mg} \quad \mathrm{Na}_{2} \mathrm{MoO}_{4} \cdot 2 \mathrm{H}_{2} \mathrm{O}, 30 \mathrm{mg}$ $\mathrm{CuSO}_{4} \cdot 5 \mathrm{H}_{2} \mathrm{O}$ and $30 \mathrm{mg}$ of KI. Thiourea $(10 \mathrm{mg} / \mathrm{l})$ was added to inhibit nitrification. The $\mathrm{pH}$ of the salt solution was adjusted to 7.2 and then sterilized, where the phosphorus components of the solution was sterilized separately. After sterilization, the two solutions were allowed to cool, and were then mixed together.

In the batch experiments, different concentrations of acetate, propionate and ammonia were tested for the two systems and different feeding regimens were used. For the acetate reactor: the acetate concentrations tested with 1.4 $\mathrm{N}$-mmol/l of ammonia were $15 \mathrm{C}-\mathrm{mmol} / \mathrm{l}, 30 \mathrm{C}-\mathrm{mmol} / \mathrm{l}$ and $60 \mathrm{C}-\mathrm{mmol} / \mathrm{l}$. The ammonia concentrations with 30 $\mathrm{Cmmol} / \mathrm{l}$ of acetate: $0.7 \mathrm{~N}-\mathrm{mmol} / \mathrm{l}, 1.4 \mathrm{~N}-\mathrm{mmol} / \mathrm{l}$ and 2.8 $\mathrm{N}$-mmol/l. The feeding regimen was tested by supplying multiple pulses of $60 \mathrm{C}-\mathrm{mmol} / \mathrm{l}$ of acetate (3 tests with 3 pulses and 1 test with 4 pulses), where $0.7 \mathrm{~N}$-mmol/l of ammonia was also added in the first pulse for these tests. Two more assays were performed by supplying a $30 \mathrm{C}-$ $\mathrm{mmol} / \mathrm{l}$ of propionate and a mixture of acetate and propionate with $15 \mathrm{C}-\mathrm{mmol} / \mathrm{l}$ each. The batch tests performed in the propionate system analyzed the effect of different concentrations of propionate $(30 \mathrm{C}-\mathrm{mmol} / \mathrm{l}, 60 \mathrm{C}-\mathrm{mmol} /$ l, $90 \mathrm{C}-\mathrm{mmol} / \mathrm{l}$ and $120 \mathrm{C}-\mathrm{mmol} / \mathrm{l}$ ) with $1.4 \mathrm{~N}-\mathrm{mmol} / \mathrm{l}$ of ammonia and the use of acetate (in one pulse of $30 \mathrm{C}$ $\mathrm{mmol} / \mathrm{l}$ and mixed with propionate, $15 \mathrm{C}-\mathrm{mmol} / \mathrm{l}$ each). In total, 18 batch tests were performed with the two systems.

\section{Analytical techniques}

Cell dry weight was determined as volatile suspended solids (VSS), according to Standard Methods [28]. Acetate and propionate were analyzed by HPLC using a BioRad Aminex HPX-87H column, with $0.01 \mathrm{~N}$ sulphuric acid as eluent, an elution rate of $0.6 \mathrm{ml} / \mathrm{min}$ and an operating temperature of $50^{\circ} \mathrm{C}$. A UV detector (Merck) set at $210 \mathrm{~nm}$ was used. Prior to injection, samples were filtered using a $0.2 \mu \mathrm{m}$ membrane. PHA were determined by GC after acidic estherification (see $[4,11]$ for details). Ammonia was analyzed using an ammonia gas sensing combination electrode (ThermoOrion 9512).

\section{Parameter estimation}

The kinetic parameters were estimated by non-linear weighted least-squares (MATLAB's lsqnonlin function) using the Levenberg-Marquardt algorithm. The program minimized the root mean squared error:

$$
r m s e=\sqrt{\frac{\mathbf{e}^{T} \cdot \mathbf{e}}{(n-p)}}
$$

with $\mathrm{e}$ the vector of residuals scaled by their maximum values, $n$ the number of measurements and $p$ the number of parameters to estimate.

The confidence bounds of the parameters were estimated by the approximation of the Hessian matrix, $\mathrm{H}$, by the Jacobian matrix, $\mathbf{J}$, at the minimum root mean squared error, rmse.

$$
\mathbf{H}=\mathbf{J}^{\mathrm{T}} \cdot \mathbf{J} \cdot r m s e
$$

Finally, confidence bounds, $\mathrm{CB}_{\mathrm{p}^{\prime}}$ were obtained by the estimate of standard deviations for a level of confidence of 95\%:

$$
\mathbf{C B}=\operatorname{diag}(\mathbf{H}) \cdot \mathrm{t}(1-0.95, n-p)
$$

with $t$ the t-student distribution.

For the calculation of residuals, Eqs. (4a-g) were integrated using a $4^{\text {th }} / 5^{\text {th }}$ order Runge-Kutta solver (MATLAB's ode45 function), with the obtained concentrations of acetate, propionate, $\mathrm{PHB}, \mathrm{PHV}, \mathrm{PH} 2 \mathrm{MV}$ and oxygen subtracted from the respective measured values to give the residuals.

\section{Metabolic Flux Analysis}

Metabolic Flux Analysis (MFA) is a methodology that allows the determination of a set of unknown metabolic fluxes, $\mathbf{v}_{\mathbf{n}^{\prime}}$ from a set of known fluxes, $\mathbf{v}_{\mathbf{b}}$ [29] by steadystate material balancing (here using Eqs. (1a-f)). In the present case, $\mathbf{v}_{\mathbf{b}}$ is composed of the modelled fluxes:

$$
\mathbf{v}_{\mathbf{b}}=\left[R_{4}, R_{5}, R_{6}, R_{7}, R_{9}, R_{10}\right]^{\mathrm{T}}
$$

The remaining fluxes were calculated by solving the central MFA equation [29]: 


$$
\mathbf{v}_{\mathrm{n}}=-\mathrm{A}_{\mathrm{n}}{ }^{-1} \cdot \mathrm{A}_{\mathrm{b}} \cdot \mathbf{v}_{\mathrm{b}}
$$

with $\mathbf{v}_{\mathbf{n}}$ the vector of unknown fluxes,

$$
\mathbf{v}_{\mathbf{n}}=\left[R_{1}, R_{2}, R_{3}, R_{8}, R_{P H B}, R_{P H V}, R_{P H 2 M V}\right]^{\mathrm{T}}
$$

$A_{n}$ and $A_{b}$ are the corresponding stoichiometric matrices of the unknown and known fluxes respectively. Note that in this case $A_{n}$ is a square $(7 \times 7)$ matrix.

For single substrate feeding studies, either $\mathrm{R}_{1}$ or $\mathrm{R}_{2}$ equals zero, thus only 5 modelled

fluxes had to be included in $\mathbf{v}_{\mathbf{b}^{\prime}}$, being $R_{6}$ moved to $\mathbf{v}_{\mathbf{n}}$.

\section{Flux Balance Analysis}

Flux Balance Analysis (FBA) is a method to optimize a given metabolic objective function under the constraints of steady-state material balancing of intracellular intermediates and other known biological constraints [30]. FBA was applied in this study to maximize the flux of PHA synthesis with a desired 24\% (C-mol/C-mol) propionyl$\mathrm{CoA}^{*}$ composition. Mathematically, the problem can be stated in the following way.

$$
\max _{R_{1}, R_{2}}\left(R_{P H B}+R_{P H V}+R_{P H 2 M V}\right)
$$

under the following constraints:

$$
\begin{gathered}
\mathbf{0}=\mathbf{A}_{\mathbf{n}} \cdot \mathbf{v}_{\mathbf{n}}+\mathbf{A}_{\mathbf{b}} \cdot \mathbf{v}_{\mathbf{b}} \\
\begin{array}{c}
0=R_{P H B}+R_{P H V}+ \\
(12 \mathrm{C})
\end{array} \\
R_{P H 2 M V}-0.76 \cdot R_{9}-0.24 \cdot R_{10} \\
R_{7}=0.02
\end{gathered}
$$

$$
\frac{R_{3}}{R_{2}}=0.43 \frac{R_{2}}{R_{1}+R_{2}}
$$

\begin{tabular}{|c|c|c|c|c|c|c|}
\hline \multirow{2}{*}{$\begin{array}{c}\text { Substrate } \\
\text { Culture enrichment }\end{array}$} & \multicolumn{2}{|c|}{ Acetate } & \multicolumn{2}{|c|}{ Propionate } & \multicolumn{2}{|c|}{ Mixture } \\
\hline & Acetate & $\begin{array}{c}\text { Propionat } \\
\text { e }\end{array}$ & Acetate & Propionate & Acetate & Propionate \\
\hline $\begin{array}{l}\text { Number of experiments used for } \\
\text { parameters estimation/model validation }\end{array}$ & $(7 / 3)$ & $(1 / 0)$ & $(1 / 0)$ & $(3 / 1)$ & $(1 / 0)$ & $(1 / 0)$ \\
\hline P/O ratio, d (mol-ATP/mol-NADH $\left.{ }_{2}\right)$ & $2.94 \pm 0.16$ & $1.08 \pm 0.12$ & $1.80 \pm 0.19$ & $0.94 \pm 0.04$ & $2.90 \pm 0.27$ & $1.78 \pm 0.19$ \\
\hline \multicolumn{7}{|c|}{ Maximum rates determined by metabolic model [C-mol/(C-mol.h)] } \\
\hline $\boldsymbol{R}_{6, \max }$ & - & - & - & - & $0.092 \pm 0.017$ & $0.094 \pm 0.046$ \\
\hline$R_{9, \max }$ & $0.52 \pm 0.03$ & $0.13 \pm 0.01$ & $0.033 \pm 0.005$ & $0.053 \pm 0.003$ & $0.30 \pm 0.03$ & $0.18 \pm 0.02$ \\
\hline$R_{10, \max }$ & - & - & $0.041 \pm 0.004$ & $0.052 \pm 0.003$ & $0.22 \pm 0.02$ & $0.13 \pm 0.02$ \\
\hline \multicolumn{7}{|c|}{ Maximum rates determined by MFA [C-mol/(C-mol.h)] } \\
\hline$R_{I, \max }$ & $0.73(0.01)$ & $0.21(0.02)$ & - & - & $0.33(0.07)$ & $0.22(0.05)$ \\
\hline$R_{2, \max }$ & - & - & $0.12(0.02)$ & $0.17(0.02)$ & $0.34(<0.01)$ & $0.23(<0.01)$ \\
\hline$R_{3, \max }$ & - & - & $0.049(0.005)$ & $0.074(0.005)$ & $0.074(0.018)$ & $0.059(0.016)$ \\
\hline$R_{6, \max }$ & $0.18(<0.01)$ & $0.07(<0.01)$ & $0.016(<0.001)$ & $0.021(0.003)$ & - & - \\
\hline$R_{8, \max }$ & $0.24(0.02)$ & $0.11(<0.01)$ & $0.094(0.006)$ & $0.13(0.02)$ & $0.19(<0.01)$ & $0.22(0.01)$ \\
\hline$R_{P H B, \max }$ & $0.52(0.03)$ & $0.13(0.01)$ & $0.011(0.003)$ & $0.018(0.001)$ & $0.14(0.02)$ & $0.090(0.005)$ \\
\hline$R_{P H V, \max }$ & - & - & $0.035(0.006)$ & $0.086(0.006)$ & $0.26(0.03)$ & $0.22(0.03)$ \\
\hline$R_{P H 2 M V, \max }$ & - & - & $0.026(0.003)$ & - & $0.12(0.01)$ & - \\
\hline
\end{tabular}

Constraint (12c) defines the desired monomeric composition of the final polymer. Constraint (12d) states that the cells are not growing during the PHA synthesis phase. The maintenance flux (Eq. 12e) was set to a known flux (see Table 4). Constraint (12f) was established on the basis of experimental observations and discussed later in the results section. FBA problems are normally solved

Table 4: Parameters estimation results of acetate and propionate enriched cultures for the different feeding conditions

The confidence bounds in parenthesis were obtained by error propagation of the metabolic model parameters $f_{P H A, \max }=2.47 \mathrm{C}-\mathrm{mmol} / \mathrm{C}-\mathrm{mmol}[\mathrm{I0}] ; \mathrm{a}=3.85$ (dimensionless) [10]; $m_{\text {ATP }}=0.02 \mathrm{~mol}-\mathrm{ATP} /(\mathrm{C}-\mathrm{mol} . \mathrm{h})[39] ; K_{S}=0.062 \mathrm{C}-\mathrm{mmol} / \mathrm{l}[39] ; K_{\mathrm{N}}=0.56 \mathrm{~N}-$ $\mathrm{mmol} / \mathrm{l}[40]$ 
through linear programming using the simplex algorithm. In our case, constraint (12f) is a nonlinear equation, thus a nonlinear function solver was adopted. The MATLAB function 'fmincon' based on the quasi-Newton optimization algorithm was adopted to solve the system (12a-f).

\section{Results and discussion Estimation of the kinetic parameters}

The data of 18 batch experiments were used for model calibration and validation. In mixed microbial cultures there is a diversity of organisms with different phenotypes competing for the nutrients in the medium. The observed metabolic activity is thus an "average" metabolic activity over all organisms present in the culture. This metabolic activity is obviously highly dependent on the culture enrichment regimen and also on the VFA feeding strategy. As such, the parameter estimation was done separately for the following 6 distinct experimental conditions:

1. Acetate enriched culture fed with acetate.

2. Acetate enriched culture fed with propionate.

3. Acetate enriched culture fed with mixtures of acetate and propionate.

4. Propionate enriched culture fed with acetate.

5. Propionate enriched culture fed with propionate.

6. Propionate enriched culture fed with mixtures of acetate and propionate.

The estimated parameter values and respective 95\% confidence limits are compiled in Table 4 . Note that the estimated confidence bounds are generally quite low, denoting the high sensitivity of residuals to parameters and the high statistical confidence of the estimated parameter values.

Figures 2, 3, 4, 5, 6, 7 compare model predictions and respective measurements for the data of the 18 experiments used in this study. The modelling results for acetate enriched cultures fed with acetate (7 calibration experiments and 3 validation experiments) and propionate enriched culture fed with propionate ( 3 calibration experiments and 1 validation experiment) are shown in Figures 2 and 3, respectively. Full symbols represent calibration points while open symbols denote validation points. These results show that the model was able to consistently describe the experimental data for a wide range of acetate and propionate feeding conditions, with regression coefficients, $\mathrm{r}^{2}$, always above 0.94 . Figures 4, 5, 6, 7 show the modelling results obtained for the remaining experiments, where both cultures were fed with substrates other than those used in the enrichment phase. In these Figures, the experimental data of the acetate, propionate, PHB, $\mathrm{PHV}, \mathrm{PH} 2 \mathrm{MV}$ and oxygen is represented by full symbols and the model prediction by full lines. A good agreement between experimental data and model predictions was obtained in all cases.

\section{PIO ratio}

The $\mathrm{P} / \mathrm{O}$ ratio is a measure of the efficiency of ATP synthesis coupled to cell respiration, indicative of the efficiency of catabolism. In acetate enriched cultures, the $\mathrm{P} / \mathrm{O}$ ratio was significantly higher than in propionate enriched cultures (Table 4). Moreover, the acetate feast and famine enrichment strategy yielded an extremely energetically effective population with a $\mathrm{P} / \mathrm{O}$ ratio close to the theoretical maximum, which is between 2-3 mol-ATP $/ \mathrm{mol}$ $\mathrm{NADH}_{2}$ in bacteria $[9,10,31-33]$. This result may suggest that the feast and famine strategy may induce not only the accumulation of intracellular reserves but also the optimization of the global energetic efficiency of the final selected culture.

The $\mathrm{P} / \mathrm{O}$ ratio results in Table 4 also show that after a short term swap in substrate, the $\mathrm{P} / \mathrm{O}$ ratio is highly affected. This behaviour is common to both cultures used in the present study. When propionate is the main source of energy, the $\mathrm{P} / \mathrm{O}$ ratio is consistently much lower $(\sim 1$ in both acetate enriched and propionate enriched cultures) than when acetate is the main source of energy (2.9 and 1.8 in acetate and propionate enriched cultures, respectively). This result is also consistent with the generally lower energetic efficiency associated with the propionate enriched culture. Interestingly, when mixtures of acetate and propionate are fed to the culture, the characteristic $\mathrm{P} /$ $\mathrm{O}$ ratio of acetate prevails over that of propionate, which suggests that catabolic activity and respiration is preferentially executed through acetate metabolism (see discussion below for more detail).

A detailed study about the $\mathrm{P} / \mathrm{O}$ ratio for microbial cells using different substrates was performed by Stouthamer [34]. In this study, a range of $\mathrm{P} / \mathrm{O}$ ratios between 2.25 and 3 were found for different substrates, whereas 2.25 was the value calculated for acetate. On the other hand, Gottschalk [19] stated that P/O ratio may also vary with bacterial species and more specifically with the number of phospholylation sites they contain. In mixed cultures containing enrichments of polyphosphate and glycogen-accumulating organisms (PAO and GAO), different $\mathrm{P} / \mathrm{O}$ ratios have been reported depending on the carbon source used for culture enrichment, namely acetate and propionate. The $\mathrm{P} / \mathrm{O}$ ratio reported for acetate enriched cultures was 1.85 for PAO [35] and 1.73 for GAO [22], while in propionate enriched cultures the $\mathrm{P} / \mathrm{O}$ ratio was reported as 1.37 for PAO and 1.29 for GAO [36]. Furthermore, it was 


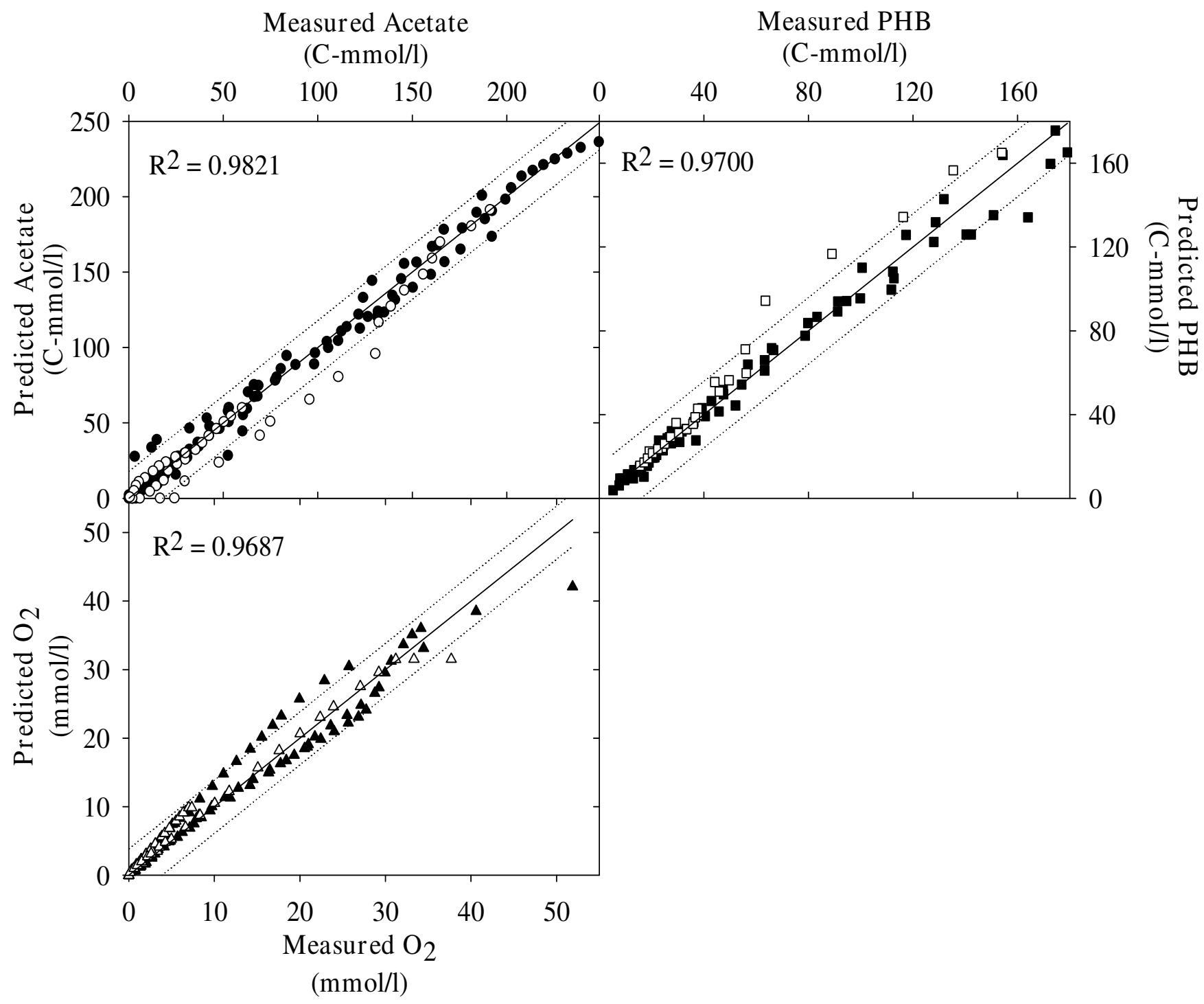

Figure 2

Modelling results. Figure shows the results obtained for acetate enriched cultures under acetate feeding conditions. Full symbols represent parameter estimation results and open symbols the model validation results. Dashed lines are the $95 \%$ confidence limits for the parameter estimation and model validation results.

observed that the mixed microbial culture fed with acetate exhibited a significantly lower $\mathrm{P} / \mathrm{O}$ ratio (1.38) at the beginning of the reactor operational period, before the acetate culture had adapted to a feast and famine regimen (data not shown). A very high $\mathrm{P} / \mathrm{O}$ ratio (2.88) was also observed for a pure culture of Cupriavidus necator (formerly Ralstonia eutropha) when imposed to the feast and famine regimen for PHA production [33]. These statements support the hypothesis that the carbon source affects the $\mathrm{P} / \mathrm{O}$ ratio and the feast and famine regimen may enable the selection of bacteria with a higher $\mathrm{P} / \mathrm{O}$ ratio, mainly in acetate enriched cultures.
Higher $\mathrm{P} / \mathrm{O}$ ratios are normally associated with lower maintenance coefficients and higher biomass/substrate and product/substrate yields. Table 5 compiles the theoretical yields for the different culture enrichment scenarios. As expected, for the same feeding conditions a higher $\mathrm{P} / \mathrm{O}$ ratio led to higher yields and lower maintenance coefficients. Within this frame, it is clear that the acetate feast and famine culture enrichment strategy produces the highest yields of cell growth and PHA formation.

\section{Metabolic flux distribution}

The calculated metabolic flux distribution (MFD) was obtained in all cases in conditions of excess of carbon 


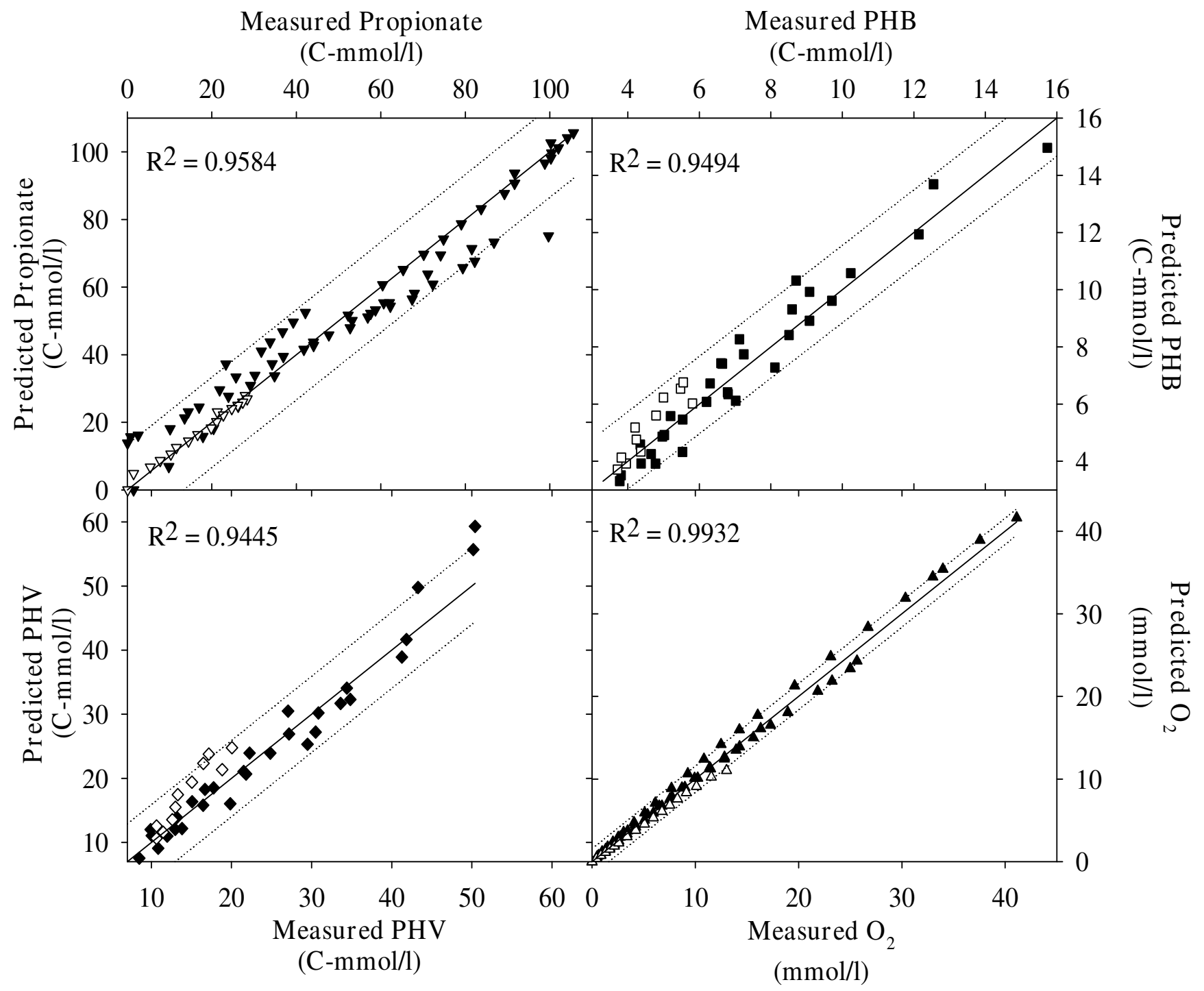

Figure 3

Modelling results. Figure shows the results obtained for propionate enriched cultures under propionate feeding conditions. Full symbols represent parameter estimation results and open symbols the model validation results. Dashed lines are the $95 \%$ confidence limits for the parameter estimation and model validation results.

source material and negligible cell growth conditions (low ammonia concentration in the medium). The results are shown in Figures $8 \mathrm{a}-\mathrm{d}$. When acetate is fed as the sole carbon source to acetate or propionate enriched cultures, the overall metabolic activity is significantly higher in the former case than in the latter case (Figure 8a). All fluxes are at least two-threefold higher in the acetate enriched culture as compared to the propionate enriched culture.

On the other hand, if propionate is fed to both cultures, all fluxes, without exception, are higher in the propionate enriched culture when compared to the acetate culture (Figure $8 \mathrm{~b}$ ). These results confirm that the substrate used for culture enrichment is more effectively metabolized by the selected culture. However, the differences between the fluxes of both cultures in Figure (8b) are markedly lower than those of Figure (8a). In particular, the total carbon uptake and corresponding PHA storage are much higher in the acetate enriched culture fed with acetate. It seems that acetate was a much more effective substrate than propionate for selective enrichment of PHA producing cultures, leading to higher quantities of PHA stored.

The simultaneous feeding of acetate and propionate to the enriched cultures is shown in Figure 8c. Acetate and propionate are taken up at similar fluxes in each culture: 0.33 


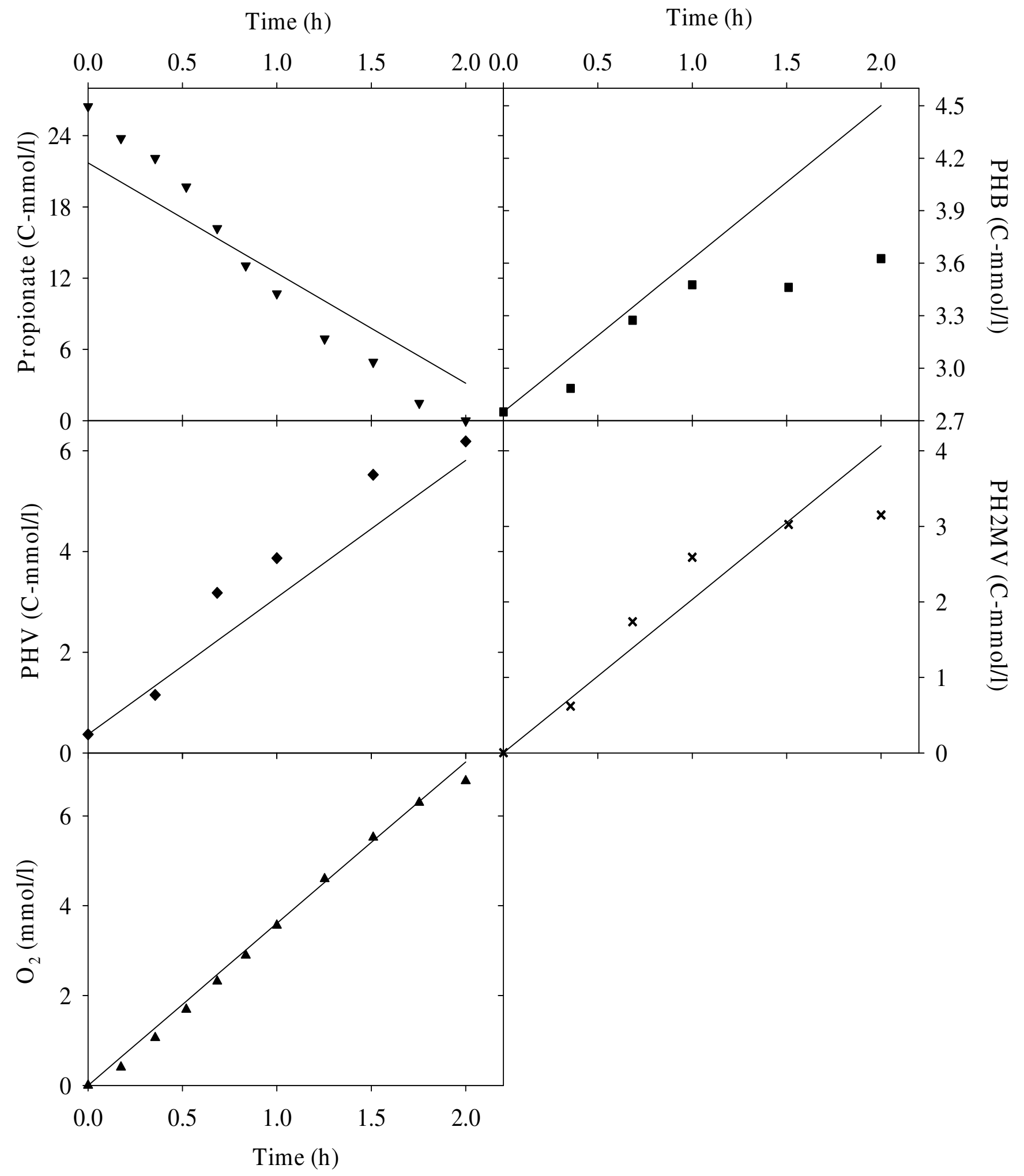

Figure 4

Modelling results. Figure shows the results obtained for acetate enriched cultures under propionate feeding conditions. Full symbols represent the experimental data and full lines the modelling results. 
Time (h)

Time (h)

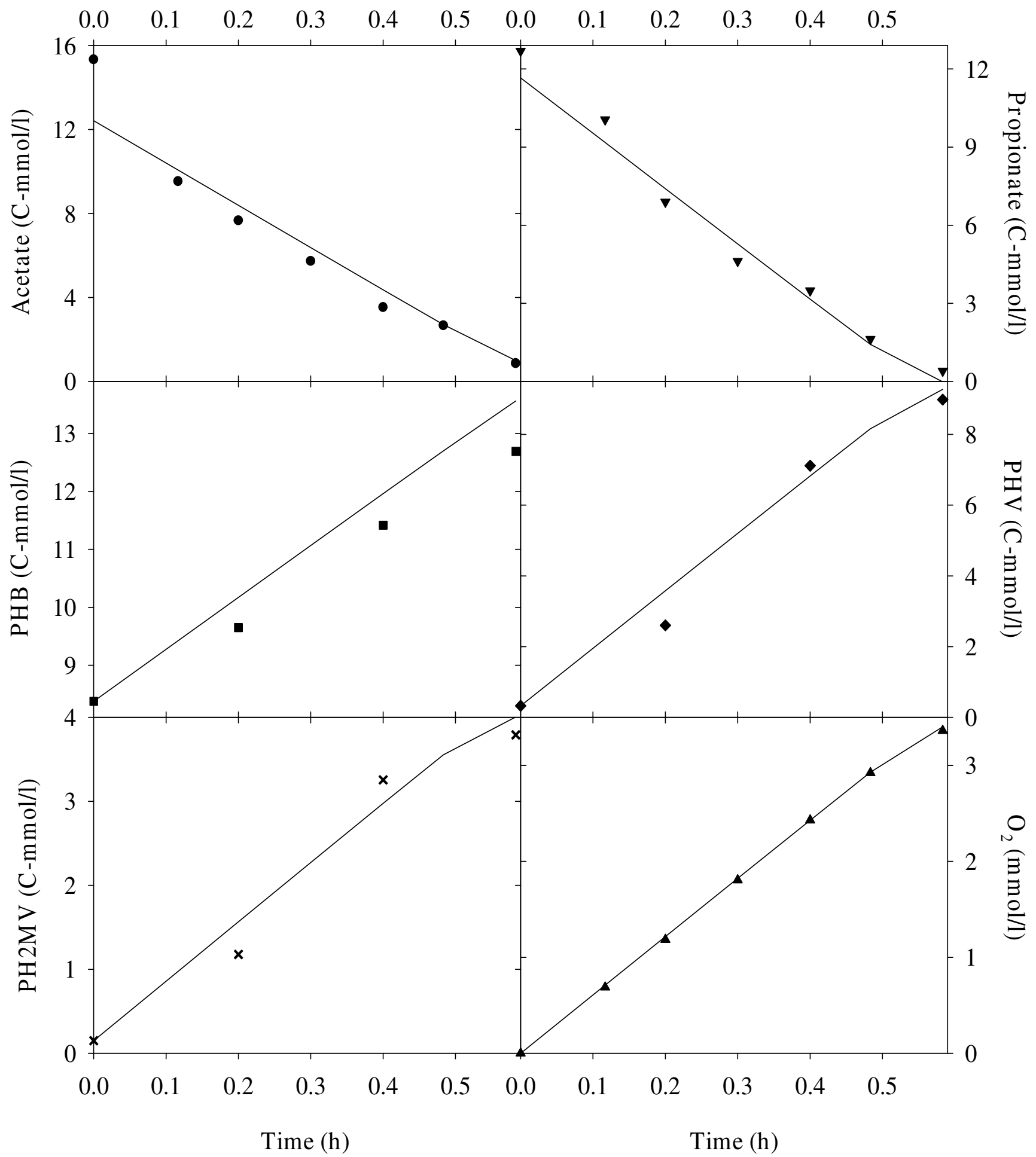

Figure 5

Modelling results. Figure shows the results obtained for acetate enriched cultures under acetate and propionate feeding conditions. Full symbols represent the experimental data and full lines the modelling results. 


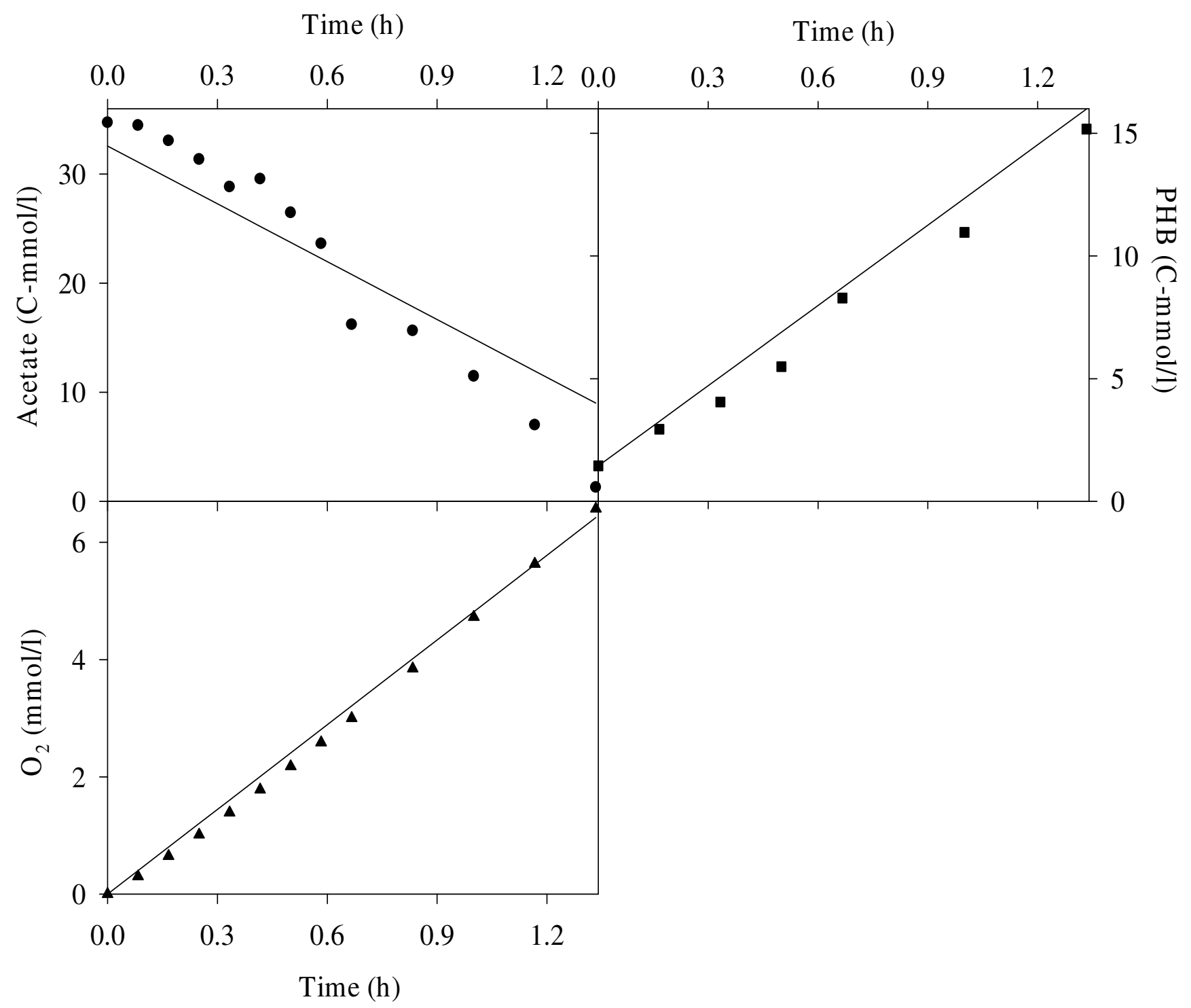

Figure 6

Modelling results. Figure shows the results obtained for propionate enriched cultures under acetate feeding conditions. Full symbols represent the experimental data and full lines the modelling results.

C-mol/(C-mol.h) of acetate and 0.34 C-mol/(C-mol.h) of propionate was taken up in acetate enriched cultures and $0.22 \mathrm{C}-\mathrm{mol} /(\mathrm{C}-\mathrm{mol} . \mathrm{h})$ of acetate and $0.23 \mathrm{C}-\mathrm{mol} /(\mathrm{C}-$ mol.h) of propionate was taken up in propionate enriched cultures. The total carbon flux is 33\% higher in acetate enriched cultures when compared to the propionate enriched cultures. The acetate enriched culture produced a total PHA flux of $0.52 \mathrm{C}-\mathrm{mol} /(\mathrm{C}-\mathrm{mol} . \mathrm{h})$, whereas the PHA flux in the propionate enriched culture is $0.31 \mathrm{C}$ $\mathrm{mol} /(\mathrm{C}-\mathrm{mol} . \mathrm{h})$ (40\% lower). However, the catabolism flux, $R_{6}$, is about the same in both cultures, suggesting that the percentage of carbon source spent for maintenance is higher for propionate enriched cultures due to the much lower $\mathrm{P} / \mathrm{O}$ ratio in propionate enriched cultures. This is further confirmed by the higher oxidative phosphorylation flux, $R_{8^{\prime}}$, for the propionate enriched culture.

It is also clear from Figures $8 \mathrm{~b}-\mathrm{c}$ that $\mathrm{PH} 2 \mathrm{MV}$ was produced in the acetate culture and not in the propionate culture, due to the fact that acetyl-CoA* and propionyl-CoA* tended to condense randomly in the acetate culture and selectively in the propionate culture. A possible explanation for this result is that selective condensation of propionyl-CoA* with acetyl-CoA* may be a property of cells acclimatized to a propionate substrate, and that the acetate culture, which was never previously exposed to propi- 


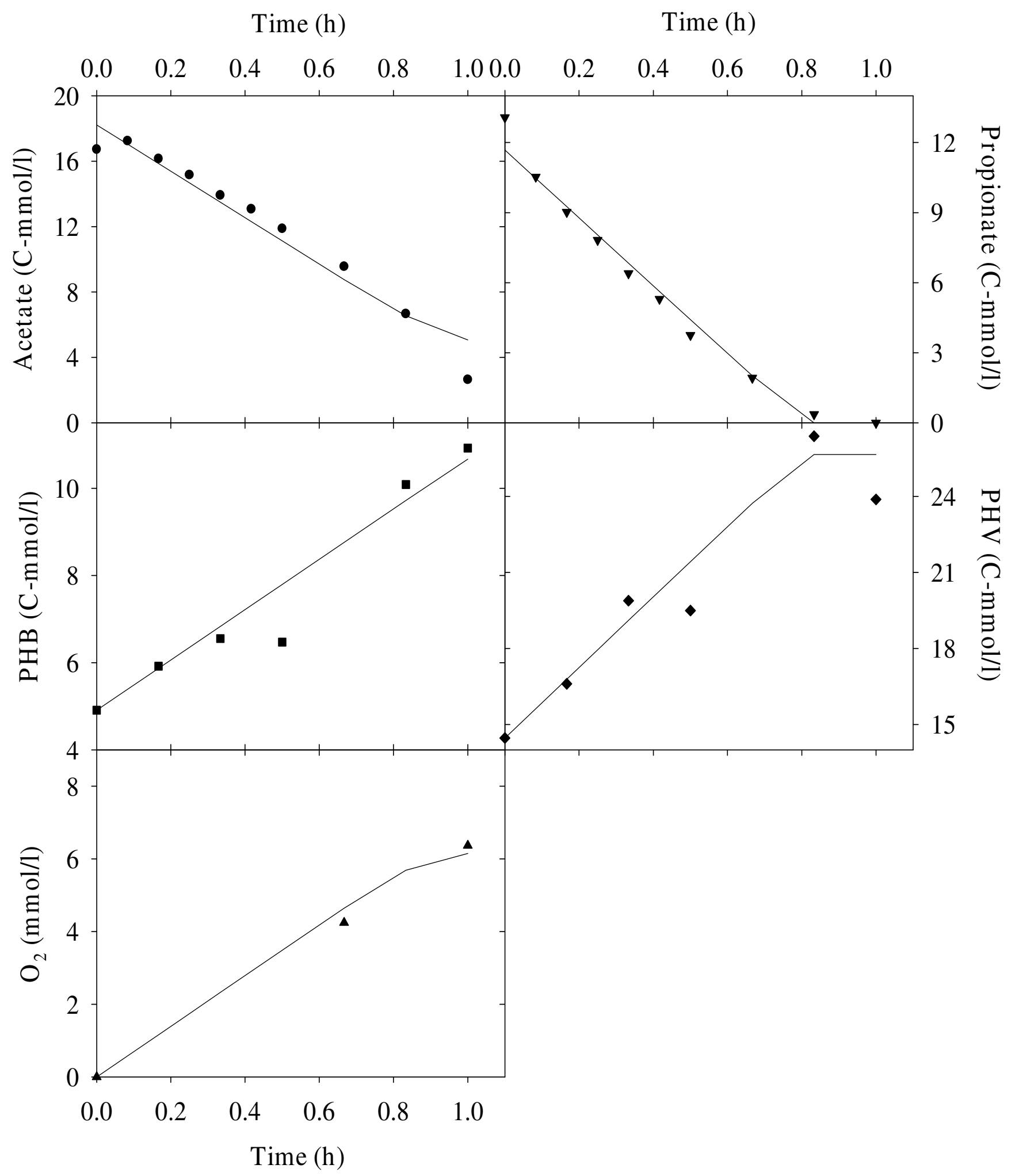

Figure 7

Modelling results. Figure shows the results obtained for propionate enriched cultures under acetate and propionate feeding conditions. Full symbols represent the experimental data and full lines the modelling results. 
Table 5: Yields and maintenance coefficients for acetate and propionate enriched cultures for the different feeding conditions

\begin{tabular}{|c|c|c|c|c|c|c|}
\hline Cultures enrichment & & Acetate & & & Propionate & \\
\hline Substrates & Acetate & Propionate & Mixture & Acetate & Propionate & Mixture \\
\hline$Y_{X, A c / S}$ & $0.66(0.01)$ & - & $0.72(0.03)$ & $0.52(0.03)$ & - & $0.50(0.10)$ \\
\hline$Y_{X, \text { Prop/S }}$ & - & $0.56(0.04)$ & $0.78(0.02)$ & - & $0.51(0.02)$ & $0.57(0.10)$ \\
\hline$Y_{P H B / S}$ & $0.76(0.01)$ & $0.75(0.02)$ & $0.84(0.01)$ & $0.68(0.02)$ & $0.71(0.01)$ & $0.71(0.06)$ \\
\hline$Y_{P H V / S}$ & - & $0.73(0.02)$ & $0.80(0.01)$ & - & $0.70(0.01)$ & $0.69(0.06)$ \\
\hline$Y_{P H 2 M V / S}$ & - & $0.72(0.02)$ & $0.78(0.01)$ & - & - & - \\
\hline$m_{S}$ & $0.0037(0.0002)$ & $0.0091(0.0010)$ & $0.0034(0.0003)$ & $0.0065(0.0008)$ & $0.011(0.001)$ & $0.0079(0.0025)$ \\
\hline$Y_{X, A c / 02}$ & $1.90(0.11)$ & - & $2.00(0.19)$ & $1.09(0.13)$ & - & $0.86(0.40)$ \\
\hline$Y_{X, \text { ProplO2 }}^{A, A C l}$ & - & $0.93(0.12)$ & $2.56(0.26)$ & - & $0.79(0.04)$ & $\mathrm{I} .10(0.5 \mathrm{I})$ \\
\hline$Y_{P H B / O 2}$ & $5.06(0.30)$ & $2.28(0.29)$ & $5.83(0.58)$ & $2.92(0.36)$ & $1.94(0.10)$ & $2.51(1.16)$ \\
\hline$Y_{P H V I O 2}$ & - & $2.52(0.32)$ & $6.32(0.63)$ & - & $2.15(0.11)$ & $2.72(1.26)$ \\
\hline$Y_{P H 2 M V I O 2}$ & - & $2.7 I(0.35)$ & $6.69(0.66)$ & - & - & - \\
\hline$m_{O 2}$ & $0.0037(0.0002)$ & $0.011(0.001)$ & $0.0037(0.0003)$ & $0.0065(0.0008)$ & $0.0120(0.0006)$ & $0.0086(0.0027)$ \\
\hline
\end{tabular}

The confidence bounds of the theoretical yields were obtained by error propagation of P/O ratio $(\delta)$

onate, may have lacked the necessary enzymes or microbial population that is responsible for selective condensation.

Figure $8 \mathrm{~d}$ shows a comparison of the fluxes for some of the main reactions in the two enriched cultures during the different feeding conditions. In acetate enriched cultures, the total VFA uptake capacity obtained with acetate feeding (0.73 C-mol/(C-mol.h)) was approximately equal to the sum of acetate and propionate uptake, when the substrates were fed simultaneously. Propionate enriched cultures showed a completely different behaviour. The total carbon uptake observed when both substrates were fed to this culture was approximately double that of the propionate or acetate uptake when the substrates were fed individually. A similar trend can be observed for the oxygen consumption flux and total PHA production flux, where the total flux in the propionate culture is approximately equal to the individual acetate and propionate fluxes combined, while the mixed substrate feed exhibited similar fluxes to the case of acetate feeding in the acetate culture. The mixed substrate feed had a synergistic effect on the individual metabolism of both substrates: both studied cultures were more able to take up propionate and convert it into PHA with a combined acetate/propionate feeding. This increase in VFA uptake was also observed in a culture adapted to a mixture of acetate, propionate and lactic acid [16]. A possible explanation for this result is that the conversion of propionyl-CoA to acetyl-CoA is the rate limiting step for PHV synthesis using only propionate. The effect of this limitation on VFA uptake rate is attenuated when acetate is also fed, because the requirement of propionyl-CoA driven to acetyl-CoA is lower and more propionyl-CoA can be driven for propionyl-CoA* synthesis without losing carbon in reaction, $R_{3}$.
Since the fraction of propionate per total VFA in the feed had a substantial influence on the amount of propionate uptake driven to acetyl-CoA, a relationship was established based on these two fractions, for each mixed culture under either a propionate or a mixed propionate-acetate feed (see Eq. 12f). The linear regression coefficient, $r^{2}$, for this equation was found to be 0.98 , based on the data presented in Table 4 . It is clear that an increase in the propionate fraction fed to the mixed cultures leads to an increase in the amount of propionate converted through propionyl-CoA to acetyl-CoA. Thus, there was a higher requirement for acetyl-CoA production when the relative acetate uptake rate is lower. This supports the hypothesis discussed above, where it was proposed that energy generation through the catabolic activity of the cells are preferentially executed through acetyl-CoA.

Overall, the results from the tests with the simultaneous feeding of acetate and propionate again corroborate the hypothesis that acetate was a much more effective substrate than propionate for the selection of an optimal PHA producing culture through enrichment via the feast and famine regimen.

\section{Flux balance analysis}

From the results presented above, it is clear that acetate is a superior substrate to propionate for the enrichment of a microbial culture performing PHA production under the feast and famine regimen, due to the higher oxidative phosphorylation efficiency with this carbon source, resulting in higher PHA productivity. It is also clear that the feeding of both acetate and propionate carbon sources simultaneously resulted in either a similar or higher total PHA productivity, with a higher diversity of 3HA monomers as compared to the feeding of a single substrate. 

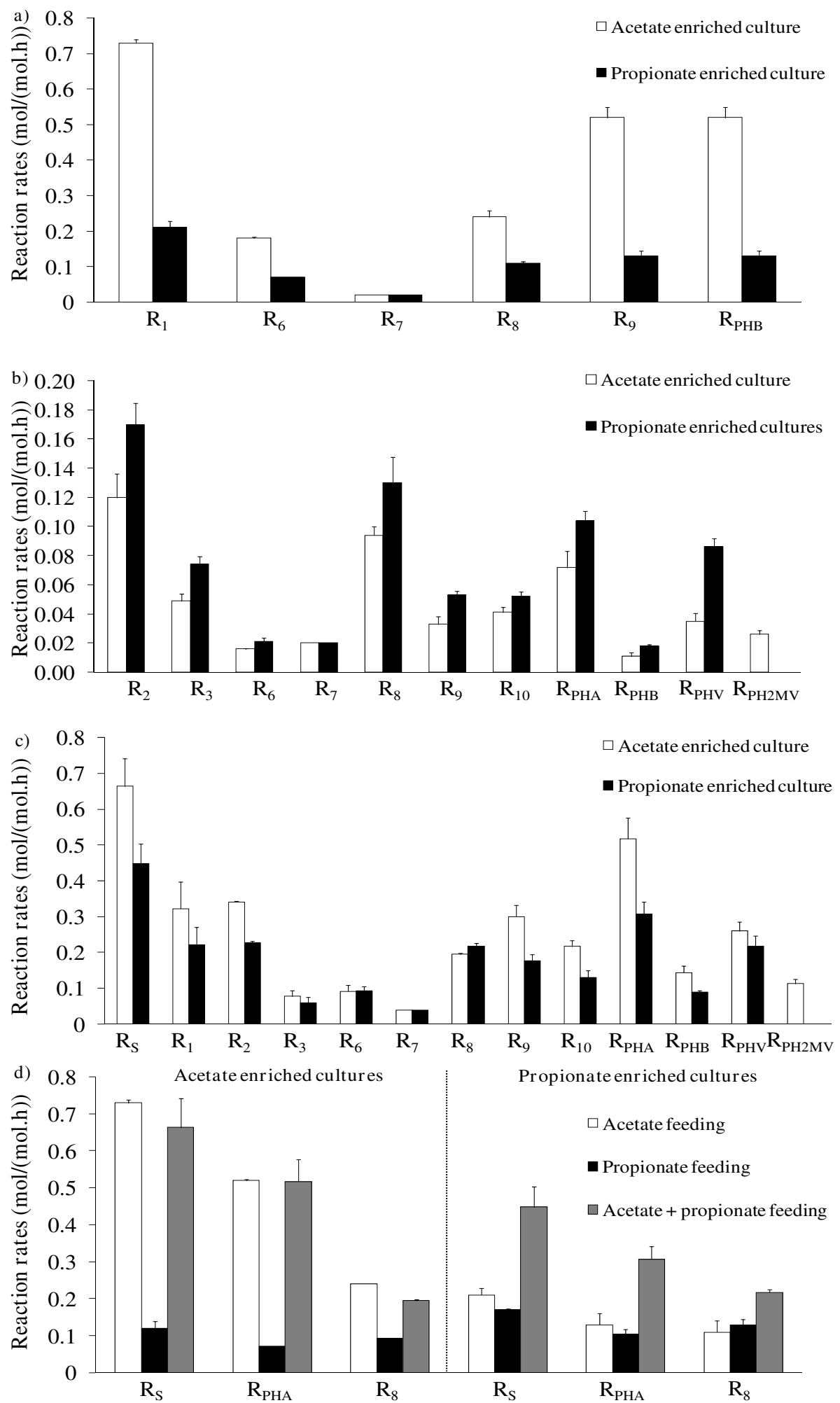

Figure 8

MFD results. Figure shows MFD results for acetate (a), propionate (b) and acetate + propionate (c) feeding using acetate ( $\square$ ) and propionate $(\square)$ enriched cultures. Figure $8 \mathrm{~d}$ presents MFD results of the main substrates uptake, PHA and oxidative phosphorylation fluxes for the two enriched cultures. 
Thus, in PHA producing systems where the microbial culture enrichment and PHA production phases are separated, it is desirable to enrich the microbial culture using acetate, while feeding a combination of acetate and propionate in the PHA production phase.

During the PHA production phase, it is possible to control the final PHA polymer composition through manipulating the acetate and propionate feeding fractions, using the metabolic model presented in this study. It was stated that a PHA copolymer with $15-20 \mathrm{~mol} \%$ of propionyl-CoA* (18-24 C-mol \%) is desired for improved toughness of the final polymer [18]. Assuming this propionyl-CoA* content as a constraint, the optimal feeding strategy required to obtain this copolymer was defined by performing FBA on the acetate enriched culture, where Eq. $12 \mathrm{f}$ was also used as a constraint. The FBA results are presented in Figure 9. These results show that the maximum PHA production rate with the desired propionyl-CoA* content is obtained when the propionate uptake rate is below the maximum calculated from parameter estimation (0.34 C-mol/(C-mol.h)). The limitation of the propionate uptake rate can be achieved by ensuring that the feeding rate of propionate is equal to the desired propionate uptake rate during reactor operation. When the process was operated at the maximum propionate uptake rate, the obtained propionyl-CoA* content in the PHA polymer was about $42 \%$.

\section{Metabolic comparison of PHA production by pure and mixed cultures}

The process efficiency for PHA production by mixed microbial cultures was evaluated through a comparison of the metabolic fluxes for the PHA production process by pure cultures of microorganisms using the same carbon substrates (i.e. acetate and propionate). In the present study using mixed microbial cultures, the biomass growth was assumed to be negligible based on the very low feed concentration of ammonia. In the pure culture studies

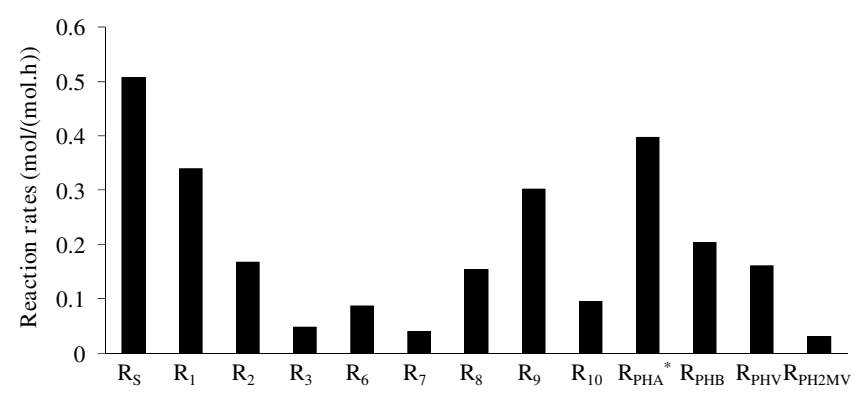

Figure 9

FBA results. Figure shows FBA results obtained by maximizing the PHA flux (RPHA*) with a propionyl-CoA* content of $24 \%$ (C-mol basis).
$[18,37]$, the maximum carbon fraction used for biomass growth was less than $15 \%$, providing a good basis for comparison with the present study. MFA was performed for a pure culture of Cupriavidus necator using acetate as the sole carbon source [37]. As shown in Table 6, the acetate uptake rate is higher for mixed microbial cultures enriched on acetate $(0.73 \mathrm{C}$-mol/(C-mol.h $)$ ) than for pure cultures $(0.44 \mathrm{C}-\mathrm{mol} /(\mathrm{C}-\mathrm{mol} . \mathrm{h}))$. However, the acetate uptake rate in the cultures enriched on propionate is half of the rate observed in pure cultures $(0.21 \mathrm{C}-\mathrm{mol} /(\mathrm{C}-$ mol.h)). The fluxes for $\mathrm{CO}_{2}$ formation and $\mathrm{O}_{2}$ consumption are slightly higher for pure cultures. These differences could be explained by the additional energetic consumption for cell growth in pure cultures that is negligible in both mixed cultures. The fraction of carbon driven for PHB is significantly higher for both mixed cultures. This is likely due to the very high $\mathrm{P} / \mathrm{O}$ ratio observed, especially in the mixed culture fed with acetate and, hence, a more efficient utilization of the carbon supply is expected.

For the case of a Cupriavidus necator culture fed with a mixture of acetate and propionate [18], the VFA uptake kinetics were observed to be different from the mixed microbial cultures enriched with either acetate or propionate, despite the fact that the initial fraction of acetate and propionate were the same in all cases $50: 50$ on a C-mol basis). The VFA uptake rate observed in the acetate enriched culture is much higher than in pure cultures (about twofold), whereas, the propionate enriched culture has only a slightly higher VFA uptake rate as compared to the pure cultures. The PHA production fluxes were observed to be much higher for the cases of the mixed cultures (78.0 and 68.6\%), as compared to the pure culture $(41.2 \%)$, during the feeding of a mixture of acetate and propionate. Additionally, despite the fact that the relative propionate uptake rate is higher in pure cultures $(63 \%)$ than in mixed cultures $(51 \%)$, the fraction of propionyl-CoA driven to propionyl-CoA* is significantly lower ( $86 \%$ lower). This is due to the fact that a large portion of the propionate fed to Cupriavidus necator was first converted to acetyl-CoA prior to PHA production, unlike the mixed culture cases. This metabolic pathway causes a large loss of $\mathrm{CO}_{2}$ by the pure culture, thus lowering the PHA production efficiency. Furthermore, a large fraction of this propionyl-CoA converted to acetyl-CoA was then used for cell catabolism, contributing to the much higher carbon flux observed in the case of the pure culture as compared to the mixed cultures.

It should be noted that the carbon source used in pure culture studies $[18,37]$ was neither acetate nor propionate, but mixtures of yeast extract, meat extract and peptone. This could be one explanation for the inferior VFA uptake and PHA production results observed in these studies as compared to the present study, since the culture may 
Table 6: Fluxes distribution for acetate and acetate + propionate feeding between mixed microbial cultures and Cupriavidus necator

\begin{tabular}{|c|c|c|c|c|c|c|}
\hline \multirow{2}{*}{$\begin{array}{c}\text { Feeding } \\
\text { Culture enrichment }\end{array}$} & \multicolumn{3}{|c|}{ Acetate } & \multicolumn{3}{|c|}{ Acetate + Propionate } \\
\hline & Acetate & Propionate & \multirow[b]{2}{*}{ Cupriavidus necator [37] } & \multirow{2}{*}{\multicolumn{2}{|c|}{ 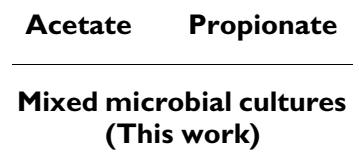 }} & \multirow[b]{2}{*}{ Cupriavidus necator $[18]$} \\
\hline \multirow[t]{2}{*}{ Cultures } & \multicolumn{2}{|c|}{$\begin{array}{l}\text { Mixed microbial cultures } \\
\text { (This work) }\end{array}$} & & & & \\
\hline & \multicolumn{6}{|c|}{ Substrate uptake rate (C-mol/(C-mol.h)) } \\
\hline VFA uptake rate & 0.73 & 0.21 & $\approx 0.441$ & 0.67 & 0.45 & $\approx 0.381$ \\
\hline Acetate uptake rate & & & & 0.33 & 0.22 & $\approx 0.161$ \\
\hline \multirow[t]{2}{*}{$\begin{array}{l}\text { Propionate uptake } \\
\text { rate }\end{array}$} & - & - & - & 0.34 & 0.23 & $\approx 0.22^{\prime}$ \\
\hline & \multicolumn{6}{|c|}{ Carbon and oxygen flux distribution (\%) } \\
\hline $\begin{array}{c}\text { Carbon converted to } \\
\text { growth }\end{array}$ & 0.7 & 3.3 & 15.0 & 2.0 & 3.3 & 8.7 \\
\hline $\begin{array}{l}\text { Carbon converted to } \\
\mathrm{CO}_{2}\end{array}$ & 25.7 & 35.0 & 37.8 & 20.0 & 28.1 & 50.1 \\
\hline $\begin{array}{l}\text { Oxygen consumed } \\
\text { per carbon }\end{array}$ & 16.5 & 27.3 & 19.8 & 14.7 & 24.3 & - \\
\hline $\begin{array}{c}\text { Carbon converted to } \\
\text { PHA }\end{array}$ & 73.6 & 61.7 & 47.2 & 78.0 & 68.6 & 41.2 \\
\hline $\begin{array}{c}\text { Carbon converted to } \\
\text { Ac-CoA* }\end{array}$ & 73.6 & 61.7 & 47.2 & 45.2 & 39.5 & 36.9 \\
\hline $\begin{array}{l}\text { Carbon converted to } \\
\text { Propionyl-CoA* }\end{array}$ & - & - & - & 32.8 & 29.1 & 4.3 \\
\hline
\end{tabular}

IApproximate rates estimated from experimental results

require an adaptation period to synthesize the necessary enzymes for the metabolism of acetate and propionate carbon sources. Alternatively, it may be that the microorganisms present in the mixed cultures are more efficient PHA producers when compared to the pure cultures of Cupriavidus necator, due to the feast and famine regimen imposed. An increased VFA uptake rate was observed for a pure culture of Amaricoccus kaplicensis when the length of the famine period was increased [38].

\section{Conclusion}

This paper presents a metabolic model of PHA copolymers production in mixed microbial cultures. The model was applied to two different cultures, obtained through two distinct enrichment protocols (selected with either acetate or propionate), under different feeding conditions (fed with either a single substrate or with mixtures of the two substrates). With this model, intracellular flux distributions for the different cases were calculated. Also, feeding scenarios were optimized by FBA targeting maximal productivity of a copolymer with a desired monomeric composition. These results were benchmarked with published results of pure cultures of Cupriavidus necator. From these studies the following main conclusions may be highlighted:
- The substrate used for culture enrichment by feast and famine feeding has a high selective pressure on the organisms that compose the final culture in the sense that the organisms selected are those that most effectively metabolize the adopted substrate.

- Sludge enrichment by acetate feeding through feast and famine regimen is much more selective towards a culture with high PHA storage fluxes and yields than when propionate is used.

- The $\mathrm{P} / \mathrm{O}$ ratio is highly dependent on the substrate and the microbial culture selected. Acetate metabolism has a consistently higher $\mathrm{P} / \mathrm{O}$ ratio (close to the theoretical maximum of $3 \mathrm{~mol}-\mathrm{ATP} / \mathrm{mol}-\mathrm{NADH}_{2}$ ) than propionate metabolism.

- MFA studies suggest that when mixtures of acetate and propionate are fed to the cultures, the catabolic activity is primarily guaranteed through acetate uptake. Coherently, the acetate $\mathrm{P} / \mathrm{O}$ ratio tends to prevail over that of propionate when both substrates are fed simultaneously.

- The application of FBA targeting the optimization of the PHA synthesis flux with a $24 \%$ (C-mol/C-mol) propionyl$\mathrm{CoA}^{*}$ composition revealed that acetate should be fed in 
excess whereas the feeding of propionate should be limited to $0.17 \mathrm{C}-\mathrm{mol} /(\mathrm{C}-\mathrm{mol} . \mathrm{h})$. Under excess of both substrates the final propionyl-CoA* content is around $42 \%$ (C-mol/C-mol).

- By comparing metabolic fluxes between mixed and pure cultures of Cupriavidus necator it can be concluded that the PHA production process by mixed microbial cultures has the potential to be comparable or even more favourable than what is achieved by pure cultures.

Although the metabolic characterization of mixed cultures was shown in this study to be highly favourable and promising, it should be noted that these results were obtained under ammonia limitation, thus, with conditions where cells growth is negligible. The compatibility of high cell growth rates with high PHA synthesis fluxes in mixed cultures is still not undoubtedly demonstrated in the literature.

\section{Abbreviations}

Acronyms: ASM3: activated sludge model No. 3; DO: dissolved oxygen; FBA: flux balance analysis; GAO: glycogenaccumulating organisms; 3HA: 3-hydroxyalkanoate; 3HB: 3-hydroxybutyrate; 3H2MB: 3-hydroxy-2-methylbutyrate; 3H2MV: 3-hydroxy-2-methylvalerate; 3HV: 3-hydroxyvalerate; HRT: hydraulic retention time; MFA: metabolic flux analysis; MFD: metabolic flux distribution; PAO: polyphosphate-accumulating organisms; PHA: polyhydroxyalkanoates; PHB: poly(3-hydroxybutyrate); PHV: poly(3-hydroxyvalerate); PH2MV: poly(3-hydroxy-2methylvalerate); $\mathbf{P} / \mathrm{O}$ ratio: ATP synthesis/oxygen consumption ratio; rmse: root mean squared error; SBR: sequencing batch reactor; SRT: sludge retention time; TCA: tricarboxylic acid cycle; VFA: volatile fatty acids; VSS: volatile suspended solids. Symbols: Ac: acetate/acetate concentration (C-mmol/l); $\mathbf{f}_{\mathbf{i}}$ : intracellular PHA, PHB, PHV and PH2MV contents (C-mol/C-mol); $\mathrm{f}_{\mathrm{PHA} \text { max }}$ : maximum intracellular PHA contents (C-mol/C-mol); $\mathbf{K}_{1}, \mathbf{K}_{2}$ : energy requirements for biomass synthesis (mol-ATP/C$\mathrm{mol}) ; \mathrm{K}_{\mathrm{S}}$ : acetate and propionate half-saturation constants $(\mathrm{C}-\mathrm{mmol} / \mathrm{l}) ; \mathrm{K}_{\mathrm{N}}$ : ammonia half-saturation constant $(\mathrm{N}-$ $\mathrm{mmol} / \mathrm{l}) ; \mathbf{m}_{\mathrm{j}}$ : maintenance on component $\mathrm{j}$ [C-mol/(Cmol.h)]; $\mathbf{m}_{\mathrm{ATP}}$ : maintenance coefficient on ATP [mol-ATP/ (C-mol.h)]; N: ammonia/ammonia concentration (Nmmol/l); $\mathbf{O}_{2}$ : oxygen/oxygen concentration (mmol/l); Prop: propionate/propionate concentration (C-mmol/l); $\boldsymbol{R}_{i}$ : specific rate of reaction on compound i [C-mol/(Cmol.h)]; $\boldsymbol{R}_{\boldsymbol{i} \text {, max }}$ : maximum specific rate of reaction on compound i [C-mol/(C-mol.h)]; S: total VFA/total VFA concentration (C-mmol/l); t: culture runtime $(\mathrm{h}) ; \mathrm{X}$ : active biomass/active biomass concentration (C-mmol/l); $y$ : fraction of propionate uptake rate in the total VFA uptake rate; $\mathrm{Y}_{\mathrm{i} / \mathrm{j}}$ : yield of component $\mathrm{i}$ on component $\mathrm{j}$ (C$\mathrm{mol} / \mathrm{C}-\mathrm{mol}) ; \alpha$ : $\mathrm{PHB}$ production saturation order con- stant (dimensionless); $\delta$ : efficiency of oxidative phosphorylation (mol-ATP/mol-NADH ${ }_{2}$ ).

\section{Authors' contributions}

All authors read and approved the final manuscript. JMLD and RO developed the software. JMLD, AO and RO participated in the model implementation. LSS and PCL performed experimental work. MAMR and RO designed and coordinated the study. JMLD, AO and RO drafted the manuscript.

\section{Acknowledgements}

This work was supported by Fundação para a Ciência e a Tecnologia (FCT) through the project $\mathrm{POCl} / \mathrm{BIO} / 55789 / 2004$ and by the Integrated Project no. 0265 15-2: Bioproduction - Sustainable Microbial and Biocatalytic Production of Advanced Functional Materials. J. Dias, A. Oehmen and L. Serafim acknowledge FCT for grants SFRH/BD/I37/4/2003, SFRH/BPD/ $4 \mid 486 / 2007$ and SFRH/BPD//4663/2003.

\section{References}

I. Dias JML, Lemos PC, Serafim LS, Oliveira C, Eiroa M, Albuquerque MGE, Ramos AM, Oliveira R, Reis MAM: Recent advances in polyhydroxyalkanoate production by mixed aerobic cultures: From the substrate to the final product. Macromolecular Bioscience 2006, 6(II):885-906.

2. Beccari M, Majone M, Massanisso P, Ramadori R: A bulking sludge with high storage response selected under intermittent feeding. Water Research 1998, 32(II):3403-3413.

3. Satoh H, Iwamoto Y, Mino T, Matsuo T: Activated sludge as a possible source of biodegradable plastic. Water Science and Technology 1998, 38(2): 103-109.

4. Serafim LS, Lemos PC, Oliveira R, Reis MAM: Optimization of polyhydroxybutyrate production by mixed cultures submitted to aerobic dynamic feeding conditions. Biotechnol Bioeng 2004, 87(2): $145-160$.

5. Albuquerque MGE, Eiroa M, Torres C, Nunes BR, Reis MAM: Strategies for the development of a side stream process for polyhydroxyalkanoate (PHA) production from sugar cane molasses. Journal of Biotechnology 2007, I 30(4):4| | -42 I.

6. Bengtsson S, Werker A, Christensson M, Welander T: Production of polyhydroxyalkanoates by activated sludge treating a paper mill wastewater. Bioresour Technol 2008, 99(3):509-516.

7. Temudo MF, Kleerebezem R, van Loosdrecht M: Influence of the $\mathrm{pH}$ on (open) mixed culture fermentation of glucose: $A$ chemostat study. Biotechnol Bioeng 2007, 98(I):69-79.

8. van Aalst-van Leeuwen MA, Pot MA, van Loosdrecht MCM, Heijnen Jj: Kinetic modeling of poly(beta-hydroxybutyrate) production and consumption by Paracoccus pantotrophus under dynamic substrate supply. Biotechnol Bioeng 1997, 55(5):773-782.

9. Third KA, Newland M, Cord-Ruwisch R: The effect of dissolved oxygen on PHB accumulation in activated sludge cultures. Biotechnol Bioeng 2003, 82(2):238-250.

10. Dias JML, Serafim LS, Lemos PC, Reis MAM, Oliveira R: Mathematical modelling of a mixed culture cultivation process for the production of polyhydroxybutyrate. Biotechnol Bioeng 2005, 92(2):209-222.

II. Lemos PC, Serafim LS, Reis MAM: Synthesis of polyhydroxyalkanoates from different short-chain fatty acids by mixed cultures submitted to aerobic dynamic feeding. Journal of Biotechnology 2006, I 22(2):226-238.

12. Lafferty M, Korsatko B, Korsatko W: Microbial Production of Poly- $\beta$-hydroxybutyric acid. Volume $6 b$. Weinheim: VCH Publishers; 1988.

13. Anderson AJ, Dawes EA: Occurrence, Metabolism, Metabolic Role, and Industrial Uses of Bacterial Polyhydroxyalkanoates. Microbiological Reviews 1990, 54(4):450-472.

14. Lee SY: Plastic bacteria? Progress and prospects for polyhydroxyalkanoate production in bacteria. Trends in Biotechnology 1996, I4(II):43I-438. 
15. Rhu DH, Lee WH, Kim JY, Choi E: Polyhydroxyalkanoate (PHA) production from waste. Water Sci Technol 2003, 48(8):22I-228.

16. Dionisi $D$, Majone M, Papa V, Beccari M: Biodegradable polymers from organic acids by using activated sludge enriched by aerobic periodic feeding. Biotechnol Bioeng 2004, 85(6):569-579.

17. Dionisi D, Carucci G, Papini MP, Riccardi C, Majone M, Carrasco F: Olive oil mill effluents as a feedstock for production of biodegradable polymers. Water Research 2005, 39(10):2076-2084.

18. Yu J, Si YT: Metabolic carbon fluxes and biosynthesis of polyhydroxyalkanoates in Ralstonia eutropha on short chain fatty acids. Biotechnol Prog 2004, 20(4): $1015-1024$.

19. Gottschalk : Bacterial metabolism. Second edition. New York: Springer-Verlag; 1986.

20. Filipe CDM, Daigger GT, Grady CPL: A metabolic model for acetate uptake under anaerobic conditions by glycogen accumulating organisms: Stoichiometry, kinetics, and the effect of pH. Biotechnol Bioeng 200I, 76(I): I7-3I.

21. Gommers PJF, Vanschie BJ, Vandijken JP, Kuenen JG: Biochemical Limits to Microbial-Growth Yields - an Analysis of Mixed Substrate Utilization. Biotechnol Bioeng 1988, 32(I):86-94.

22. Zeng RJ, van Loosdrecht MCM, Yuan ZG, Keller J: Metabolic model for glycogen-accumulating organisms in anaerobic/aerobic activated sludge systems. Biotechnol Bioeng 2003, 81(1):92-105.

23. Henze M, Harremoes P, Jansen JLC, Arvin E: Wastewater Treatment: Biological and Chemical Processes. Heidelberg: Springer; 1995.

24. Roels JA: Macroscopic Thermodynamics and the Description of Growth and Product Formation in Microorganisms. Acs Symposium Series 1983, 207:295-322.

25. Lee JH, Lim HC, Hong J: Application of nonsingular transformation to online optimal control of poly-beta-hydroxybutyrate fermentation. Journal of Biotechnology 1997, 55(3): 135-150.

26. Lee JH, Hong J, Lim HC: Experimental optimization of fedbatch culture for poly-beta-hydroxybutyric acid production. Biotechnol Bioeng 1997, 56(6):697-705.

27. Tohyama M, Patarinska T, Qiang ZW, Shimizu K: Modeling of the mixed culture and periodic control for PHB production. Biochemical Engineering Journal 2002, I0(3): $157-173$.

28. APHA: Standard methods for the examination of water and wastewater. Washington DC: American Public Health Association; 1989.

29. Stephanopoulos GN, Aristidou AA, Nielsen J: Metabolic Engineering: Principles and Methodologies. San Diego: Academic Press; 1998.

30. Edwards JS, Ibarra RU, Palsson BO: In silico predictions of Escherichia coli metabolic capabilities are consistent with experimental data. Nat Biotechnol 200I, I9(2): I25-130.

3I. Beun JJ, Paletta F, Van Loosdrecht MCM, Heijnen J]: Stoichiometry and kinetics of poly-beta-hydroxybutyrate metabolism in aerobic, slow growing, activated sludge cultures. Biotechnol Bioeng 2000, 67(4):379-389.

32. Sin G, Guisasola A, De Pauw DJW, Baeza JA, Carrera J, Vanrolleghem PA: A new approach for modelling simultaneous storage and growth processes for activated sludge systems under aerobic conditions. Biotechnol Bioeng 2005, 92(5):600-613.

33. Frigon $D$, Muyzer $G$, van Loosdrecht $M$, Raskin L: rRNA and polybeta-hydroxybutyrate dynamics in bioreactors subjected to feast and famine cycles. Appl Environ Microbiol 2006 72(4):2322-2330.

34. Stouthamer AH: Theoretical Study on Amount of ATP Required for Synthesis of Microbial Cell Material. Antonie Van Leeuwenhoek Journal of Microbiology 1973, 39(3):545-565.

35. Smolders GJF, Vandermeij J, Vanloosdrecht MCM, Heijnen JJ: Stoichiometric Model of the Aerobic Metabolism of the Biological Phosphorus Removal Process. Biotechnol Bioeng 1994, 44(7):837-848.

36. Oehmen A, Zeng RJ, Keller J, Yuan Z: Modeling the Aerobic Metabolism of Polyphosphate-Accumulating Organisms Enriched with Propionate as a Carbon Source. Water Environment Research 2007, 79(13):2477-2486.

37. Shi HD, Shiraishi M, Shimizu K: Metabolic flux analysis for biosynthesis of poly(beta-hydroxybutyric acid) in Alcaligenes eutrophus from various carbon sources. J Ferment Bioeng 1997, 84(6):579-587.

38. Aulenta F, Dionisi D, Majone M, Parisi A, Ramadori R, Tandoi V: Effect of periodic feeding in sequencing batch reactor on sub- strate uptake and storage rates by a pure culture of Amaricoccus kaplicensis. Water Research 2003, 37( I I):2764-2772.

39. Beun JJ, Dircks K, Van Loosdrecht MCM, Heijnen JJ: Poly-betahydroxybutyrate metabolism in dynamically fed mixed microbial cultures. Water Research 2002, 36(5): I I67-I I80.

40. Gujer W, Henze M, Mino T, van Loosdrecht M: Activated Sludge Model No. 3. Water Science and Technology 1999, 39(I): 183-193.
Publish with Bio Med Central and every scientist can read your work free of charge

"BioMed Central will be the most significant development for disseminating the results of biomedical research in our lifetime. "

Sir Paul Nurse, Cancer Research UK

Your research papers will be:

- available free of charge to the entire biomedical community

- peer reviewed and published immediately upon acceptance

- cited in PubMed and archived on PubMed Central

- yours - you keep the copyright 